\title{
3-3 Piezoelectric Metamaterial with Negative and Zero Poisson's Ratio for Hydrophones Applications
}

\author{
Kamran A. Khan ${ }^{1 *}$, Muhammad Ali Khan ${ }^{2}$ \\ ${ }^{1}$ Department of Aerospace Engineering, Khalifa University of Science and Technology \\ (KUST), Abu Dhabi, UAE \\ ${ }^{2}$ School of Aerospace, Transport and Manufacturing, Cranfield University, UK
}

\begin{abstract}
This study presents the micromechanical-finite-element based modeling framework to compute the electromechanical properties of the 3-3 piezoelectric metamaterial based on variants of honeycomb (HC) structure. Recent experimental study shows that 3-3 piezoelectric structures have a higher hydrostatic figure-of-merit compared to dense piezoelectric hydrophones of similar design. In this study, three kinds of three-dimensional (3D) elastically anisotropic and piezoelectrically active $\mathrm{HC}$ structures were introduced, namely, conventional HC (3D-CHC), a reentrant HC (3D-RE) and a semi-re-entrant HC (3D-SRE). The intrinsic symmetry of HC structure was utilized.Simplified mixed boundary conditions equivalent to periodic boundary conditions were recognized. Highly porous 3D finite element models of the mentioned three kinds of metamaterials were developed and the role of ligament orientation on their effective elastic, piezoelectric and dielectric properties was completely characterized. In comparison to their bulk constituent, all the 3-3 type piezoelectric HC networks exhibited an enhanced response, especially for the longitudinal poling. Longitudinally poled networks exhibited four order of magnitude increase in their hydrostatic figure of merit and one order of magnitude decrease in the acoustic impedance indicating their applicability for the design on hydrophones. Moreover, different type of auxetic behavior such as negative or zero Poisson's ratio coupled with a wide range of tunable
\end{abstract}

\footnotetext{
* Corresponding author. Tel.: +971-(02) 4018227; Fax: +971-(02) 4472442

E-mail address: kamran.khan@ kustar.ac.ae (K.A. Khan)
} 
electromechanical characteristics were obtained. Such novel 3-3 type piezoelectric HC network based cellular metamaterials have the potential to facilitate the light-weight design of unique devices for various next generation sensing or actuating applications.

Keywords: metamaterials; electromechanical properties, honey comb structures, unit cell method, piezoelectric materials, auxetic smart structures, cellular materials.

\section{Introduction}

Piezoelectric materials (PMs) due their unique electromechanical coupling characteristics are useful in wide range of actuators, sensors and devices such as ultrasound imagers, underwater transducers and echo-cardiogram [1], [2]. Various efforts have been made to enhance the electromechanical coupling properties of monolithic PMs by either adding one or more constituents [3] or by introducing the controlled porosity [4]. For example, porous PMs with enhanced electromechanical coupling properties, improved signal-to-noise ratio, lower acoustic impedance, enhanced piezoelectric charge, voltage coefficients and hydrostatic figure of merit. They are also suitable for underwater applications such as medical diagnostic devices [5] and hydrophones [6]. In fact, recent experimental study shows that porous piezoelectric structures have a higher hydrostatic figure-of-merit [7],[8] as compareto solid PZT hydrophones of similar design [2], [9]. Mechanical properties are also of great interest to use these materials in structural applications. Therefore, there is a need in various industrial applications for porous piezoelectric metamaterials with tunable properties.

Porous PMs are classified according to the spatial distribution of the porosity or its connectivity in different direction [10], which affect their piezoelectric properties [11]. The role of connectivity of the porosity on the electromechanical response was well understood from experimental studies with several porosity configurations such as enclosed porosity (i.e.,3-0 type foam) ([12]; [2]; [9]; 
[13];); fiber like porosity (i.e.,3-1 type foam) ([14]; [15]) and open-foam like porosity (i.e.,3-3 type foam) ([16]; [17]). The results demonstrated that porosity configuration and its connectivity were responsible for enhancing the electromechanical properties of PMs.

Piezoelectric metamaterials and piezoelectric architected materials (e.g., [18], [19], [20], [21]; [13]) are the potential subclass of porous PMs. It is because their microstructure in any porosity configuration (such as 3-0, 3-1 and 3-3) can be tailored to produce new innovative material designs with optimum multifunctionality required for specific applications [22].

Various analytical models were proposed to calculate electromechanical properties of PM's for simplified porosity configurations (such as 3-0 and 3-1 types) [23], [24], [25], [26]. While various finite element based micromechanical models were developed for more complex architected porosity configurations (such as3-0, 3-1 and 3-3 type) for computing their electromechanical properties [27], [28]. Results concluded that electromechanical properties of porous PMs depend on porosity shape, orientation and distribution, connectivity and poling direction ([29], [18]). Metamaterials are materials of interest due to their exciting behavior with enhanced properties. Among architected metamaterials, the passive (mechanical) response of hexagonal honeycomb (HC) configuration has been extensively studied due to its simplicity and manufacturability. Various variants of $\mathrm{HC}$ exist based on the ligament orientations that leads to produce auxetic effects with negative and zero Poisson ratio's.[30]. Extensive research has been done on passive structural response of hexagonal $\mathrm{HC}$ variants made from elastically isotropic materials demonstrating conventional and auxetic behaviors [30], [31], [32], [33]. For both 3-1 and 3-3 porosity configuration, the mechanical analysis results demonstrated that the ligament orientation dictates the hexagonal HC architecture-property relationship ([34]; [35]. For smart piezoelectric metamaterials, due to the coupled electro-mechanical nature, there is added complexity in finding 
optimum electromechanical properties. These includes the poling direction and piezoelectric anisotropic properties of the base materials. This study proposes three classes of 3D HC network based 3-3 type piezoelectric metamaterials namely, conventional HC (3DCHC), a re-entrant HC (3DRE) and a semi-re-entrant HC (3DSRE). Recent research efforts showed promising electromechanical properties for 3-1 type $\mathrm{HC}$ based piezoelectric metamaterials which motivate the present study [36].

Currently, there is no analytical model available that compute the electromechanical properties of 3-3 type HC network based piezoelectric metamaterials. Moreover, the complete understanding of their electromechanical properties and the microstructural features (such as ligament orientation and constituents' material anisotropy) is still not available. This study presents finite element models to characterize the effects of ligament orientation and constituents' material anisotropy on the electromechanical properties of three classes of 3D HC network (i.e. based on 3-3 type piezoelectric metamaterials).

\section{Micromechanical Finite Element Analysis of Piezoelectric Metamaterials}

Recently, we ([37]) developed a finite element-based homogenization framework to compute the effective mechanical properties of a periodic architectured materials based upon the properties of base materials and topological configuration in the unit cell (UC). In this study, we have extended the framework to compute the effective electromechanical properties of a periodic piezoelectric cellular architecture materials. Boundary conditions equivalent to PBC are developed and verified. Full characterization of linear electromechanical properties of piezoelectric architecture materials can be realized using this approach.

\subsection{Architecture of Piezoelectric Metamaterial}


Three classes of three dimensional HC like piezoelectric metamaterials are considered. These include conventional hexagonal HC (C-HC), a re-entrant HC (RE-HC) (which is known to generate negative Poisson's ratio) and a semi-re-entrant HC (SRE-HC) (which is constructed using alternate conventional and re-entrant layers and produces zero Poison's ratio) structure. Yang et al. [38] proposed 3D re-entrant honeycomb passive structure. This study utilizes existing 3D reentrant honeycomb and proposes new piezoelectric 3D C-HC and 3D SRE-HC.

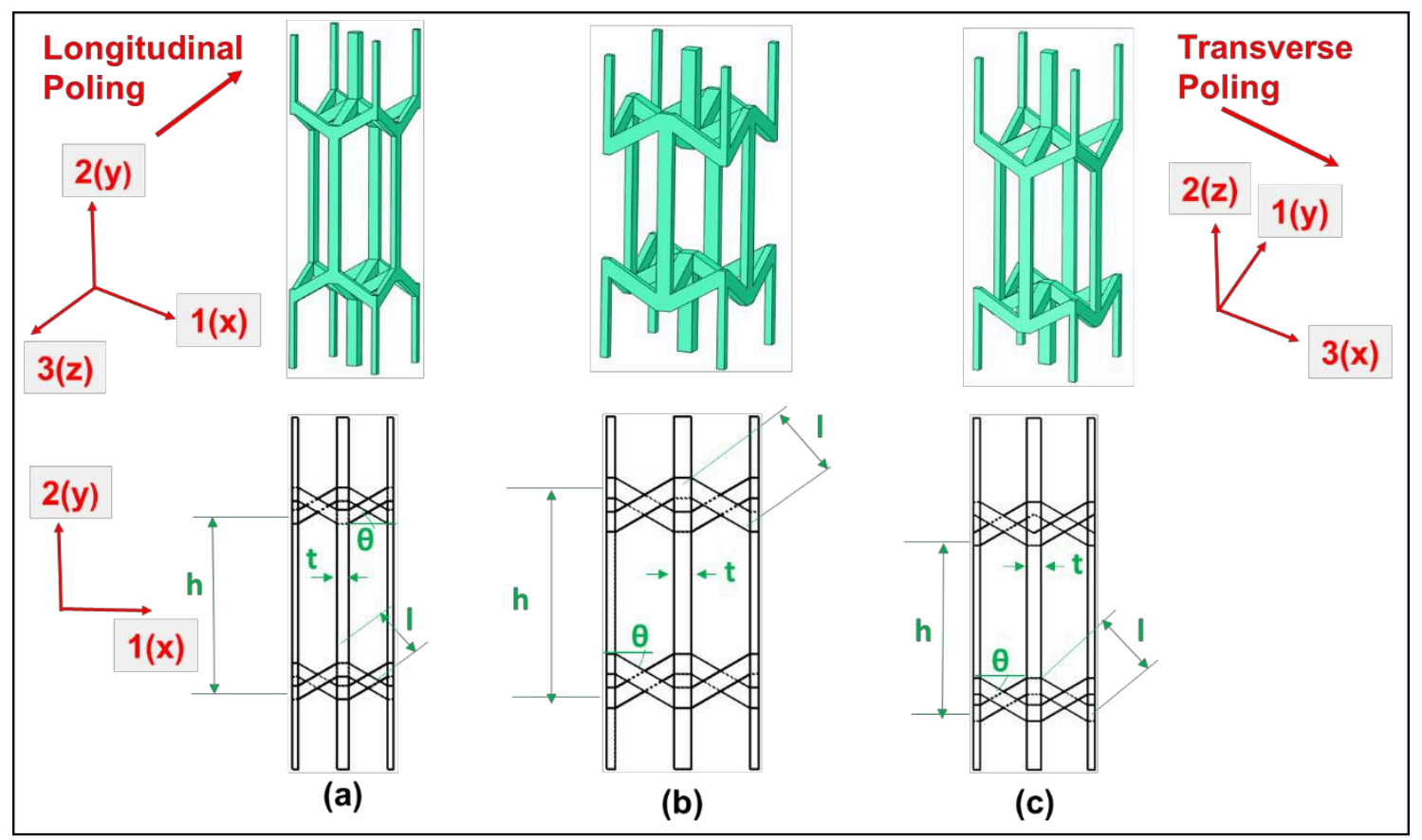

Figure 1 shows the unit cell of each architecture. These classes of HC show variety of deformation behavior and can produce positive, negative and even zero Poisson's ratio in certain configurations.

The 2D geometry of the proposed cellular structures is completely described through four parameters. The vertical rib height ' $h$ ', length of the angular rib ' 1 ', thickness of each rib ' $t$ ' and angle of the rib ' $\theta$ ' with respect to the horizontal or vertical axes. The $3 \mathrm{D}$ geometry of unit cell is extended based on these parameters [38]. We have fixed the $h=10 \mathrm{~mm}, 1=4 \mathrm{~mm}, \mathrm{t}=1 \mathrm{~mm}$ while the 
orientations

of the

ligaments

$(\theta)$

appearing

in
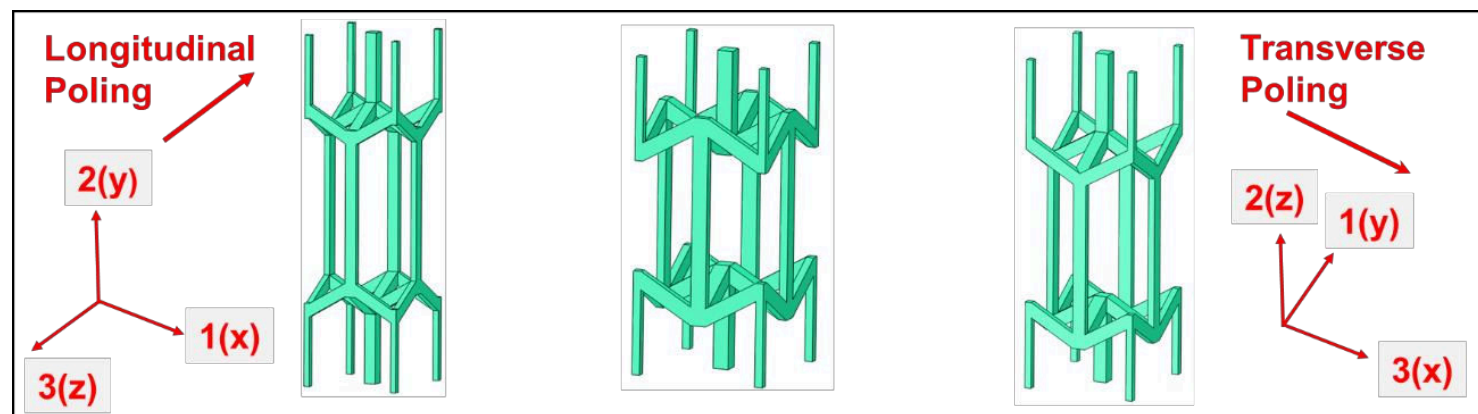

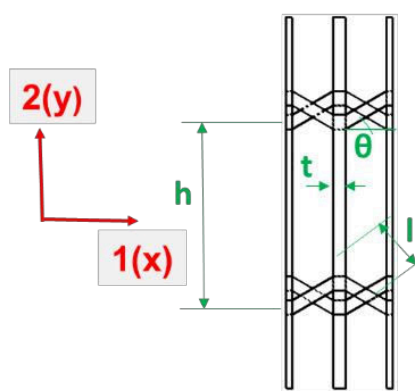

(a)

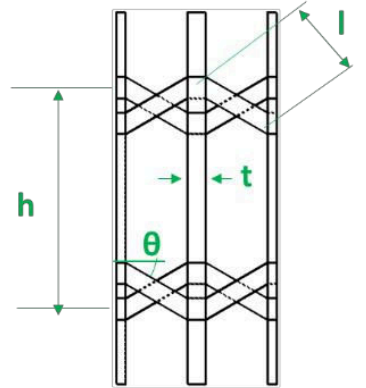

(b)

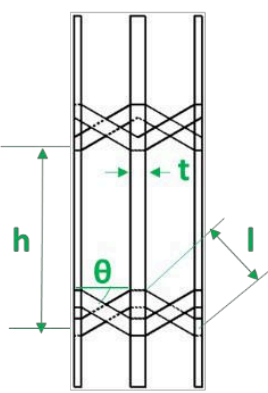

(c)

Figure 1 are varied to realize cellular structures with various auxeticity.

The ligaments of cellular solids are composed of soft piezoelectric material (PZT-5H). Two poling directions are considered and they are referred to as longitudinal (i.e. poling aligned with 
116 the axis of porosity) and transverse (i.e. poling perpendicular to the axis of porosity).

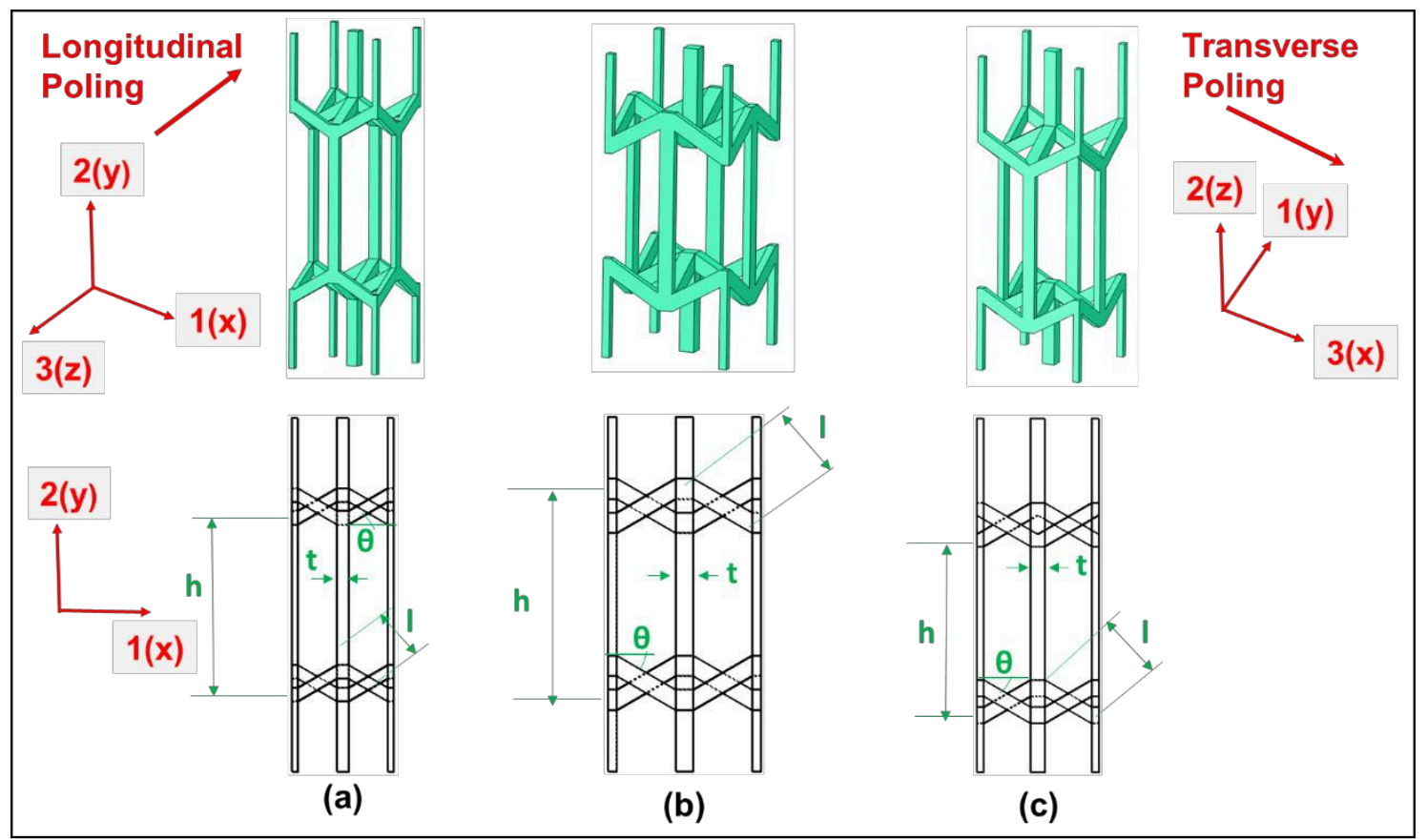

Figure 1 shows the architecture of cellular materials with the direction of poling axis and the corresponding unit cell.

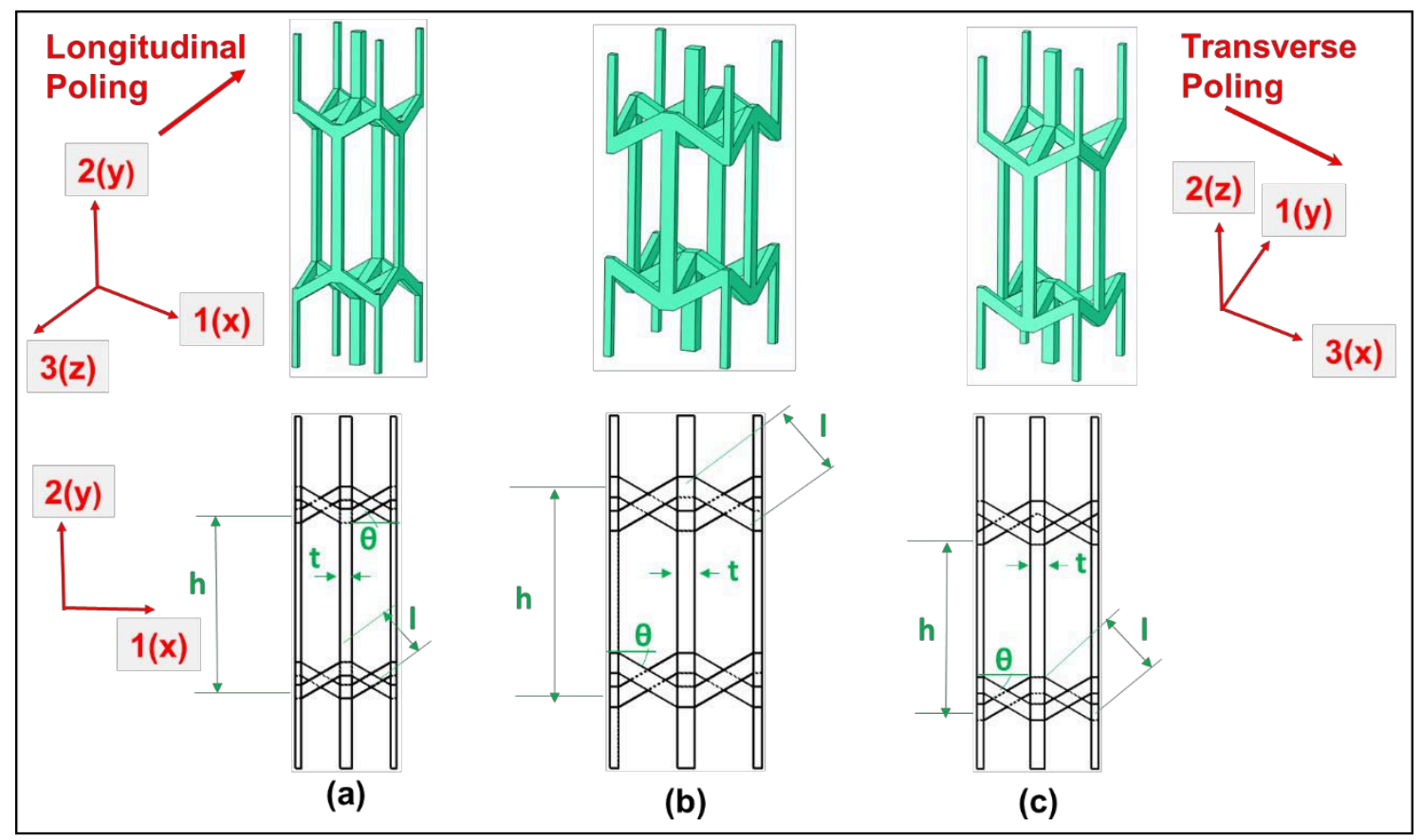

Figure 1 Piezoelectric hexagonal HC cellular networks with their unit cells a) Conventional hexagonal HC structure (CHC) b) a re-entrant HC (RE) c) semi-re-entrant HC (SRE). 


\subsection{Constitutive Model of Piezoelectric Cellular Material}

For monolithic piezoelectric material, the linearized electromechanical coupled constitutive relations are given by:

$$
\begin{aligned}
& \varepsilon_{i j}=S_{i j k l}^{E} \sigma_{k l}+d_{k i j} E_{k} \\
& D_{i}=d_{i k l} \sigma_{k l}+\kappa_{i j}^{\sigma} E_{j}
\end{aligned}
$$

where $i, j, k, l=1,2,3$. The $i, j$ and $k$ correspond respectively to $x, y$ and $z$ regular Cartesian coordinates; where to $x, y$ and $z$ can be represented as 1,2 and 3 . The $\varepsilon_{i j}, \sigma_{i j}, D_{i}$ and $E_{i}$ are the are the field variables and denote the components of the second order strain tensor, stress tensor, electric displacement vector and electric field vector, respectively. The material parameters are $S_{i j k l}^{E}, \kappa_{i j}^{\sigma}$ and $d_{i j k}$. The $S_{i j k l}^{E}$ are the components of the fourth-order compliance tensor with the superscript $E$ indicating that the values of the components of compliance tensor, $S_{i j k l}^{E}$ corresponds measurement at zero or constant electric field, $\kappa_{i j}^{\sigma}$ is the second order dielectric tensor measures at zero or constant stress and $d_{i j k}$ are the components of the piezoelectric strain tensor. The piezoelectric constants $d_{i j k}$ defined at zero stress, $d_{i j k}^{\sigma}$ at zero electric field and $d_{i j k}^{E}$ are thermodynamically identical; thus, their superscripts are not shown. Further discussion regarding the piezoelectric material behavior can be found in [39]. The Eq. (1) is applied in terms of effective stresses $\bar{\sigma}_{i j}$, strains $\bar{\varepsilon}_{i j}$, electric field $\bar{E}_{i}$ and electric displacement $\bar{D}_{i}$ for macroscopic response and to obtain effective compliance $\bar{S}_{i j k l}^{E}$, piezoelectric $\bar{d}_{k i j}$ and dielectric coefficients $\bar{\kappa}_{i j}^{\sigma}$.

$$
\begin{aligned}
& \bar{\varepsilon}_{i j}=\bar{S}_{i j k l}^{E} \bar{\sigma}_{k l}+\bar{d}_{k i j}^{E} \bar{E}_{k} \\
& \bar{D}_{i}=\bar{d}_{i k l}^{\sigma} \bar{\sigma}_{k l}+\bar{\kappa}_{i j}^{\sigma} \bar{E}_{j}
\end{aligned}
$$


Based on Eq. (2), the complete characterization of the effective electromechanical properties of piezoelectric cellular material requires quantifying 45 independent material constants, i.e., 21 elastic, 18 piezoelectric and 6 dielectric constants.

\subsection{Finite Element Models}

Finite element models of three types of HC like piezoelectric metamaterials UCs as shown in Figure 1 are generated by changing ligament orientation ranging from 30-60 degree which corresponds to a porosity values ranging from $50-85 \%$. The commercial finite element analysis software ABAQUS ${ }^{\odot}$ is used to carry out the analysis. Each ligament of the unit cell is considered to be made of soft piezoelectric material (PZT-5H). The electromechanical properties of PZT-5H are given in Table 1. Figure 2 shows the example of the unit cell of 3D HC-RE showing 6 boundary faces with respect to the axes directions. Each UC is meshed with 10-noded quadratic piezo-electric tetrahedron elements (C3D10E). Each node in the FE model has a total four degree of freedom, three displacement $\left(u_{1}, u_{2}, u_{3}\right)$ and one electrical potential $(\phi)$. To avoid rigid body 
motion under $\mathrm{e}^{1 \ldots \ldots}$ al loading, the location of arbitrary $\cdots \cdots+\mathrm{A}, \mathrm{B}$ and $\mathrm{C}$ are also shown that (a) (b)

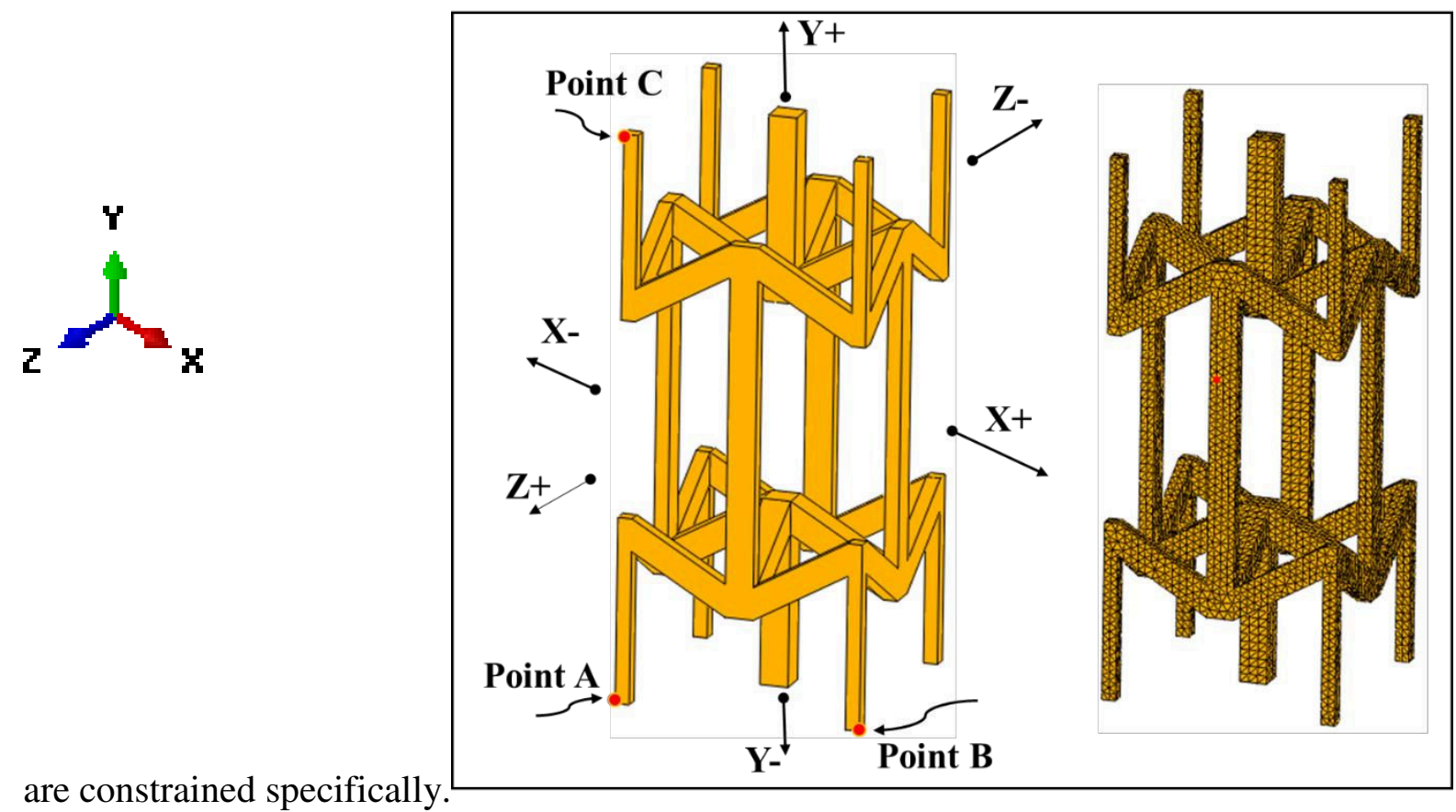

Figure 2 (a) The unit cell of 3D HC-RE showing 6 boundary faces with respect to the axes directions. (b) Meshed UC with 10-noded quadratic piezo-electric tetrahedron elements (C3D10E)

Table 1. Properties of the model piezoelectric system PZT-5H (poled in 3-direction)

\begin{tabular}{|l|ll|}
\hline Properties & PZT-5H & 164 \\
\hline$S_{11}^{E}=S_{22}^{E}\left(\mathrm{pm}^{2} / \mathrm{N}\right)$ & 16.5 & 166 \\
$S_{12}^{E}=S_{21}^{E}\left(\mathrm{pm}^{2} / \mathrm{N}\right)$ & -4.78 & \\
$S_{13}^{E}=S_{23}^{E}\left(\mathrm{pm}^{2} / \mathrm{N}\right)$ & -8.45 & 167 \\
$S_{33}^{E}\left(\mathrm{pm}^{2} / \mathrm{N}\right)$ & 20.7 & \\
$S_{44}^{E}\left(\mathrm{pm}^{2} / \mathrm{N}\right)$ & 42.6 & 168 \\
$S_{55}^{E}=S_{66}^{E}\left(\mathrm{pm}^{2} / \mathrm{N}\right)$ & 43.5 & 169 \\
$d_{15}=d_{24}(\mathrm{p} \mathrm{m} / \mathrm{V})$ & 741 & \\
$d_{31}=d_{32}(\mathrm{p} \mathrm{m} / \mathrm{V})$ & -274 & 170 \\
$d_{33}(\mathrm{pm} / \mathrm{V})$ & 593 & 171 \\
$\kappa_{11}^{\sigma} / \kappa_{0}=\kappa_{22}^{\sigma} / \kappa_{0}$ & 3130 & 172 \\
$\kappa_{33}^{\sigma} / \kappa_{0}$ & 3400 & \\
Density $(\rho)$ & $7500 \mathrm{~kg} / \mathrm{m}^{3}$ & 173 \\
Permittivity of free space $\left(\kappa_{0}\right)$ & $8.85 \times 10^{-12} \mathrm{C} / \mathrm{Vm}$ & \\
\hline
\end{tabular}


Two poling directions are considered in the analysis. The UC are assumed to be pole longitudinally (poled along the $\mathrm{z}$ axis) and transversely (poled along the $\mathrm{x}$ axis). In this study, it is assumed that every region of the finite element model is uniformly poled in one preferred direction. Poling along the $\mathrm{y}$-axis in a uniform manner is very challenging task due to the height of UC along y-axis. However, the fabrication of the cellular HCs porous networks with uniform poling of ligaments along the $\mathrm{x}$ and $\mathrm{z}$ direction has been successfully demonstrated in past [36].

\subsubsection{Boundary conditions}

The effective responses of infinitely repeating patterns of periodic architecture can be obtained by imposing Periodic Boundary Conditions (PBCs) on the UC ([40], [41], [42], [43]). PBCs give more reasonable results as compared to the ones obtained from homogeneous traction or displacement boundary conditions ([43],[45], [46]). Xia et al. [46] derived an explicit PBC for a parallelepiped UC in terms of normal average stretches and contractions $\left(c_{i}^{j}, i=j=1,2,3\right)$ of the UC model and shear deformations $\left(c_{i}^{j}, i \neq j\right)$, such that

$$
u_{i}^{j+}(\mathrm{x}, \mathrm{y}, \mathrm{z})-u_{i}^{j-}(\mathrm{x}, \mathrm{y}, \mathrm{z})=c_{i}^{j} \quad(i, j=1,2,3)
$$

where $j^{+}$means along the positive $X_{j}$ direction and $j^{-}$means along negative $X_{j}$ direction. Similarly, the periodic boundary conditions for the electric potential are given by the applied macroscopic electric field condition

$$
\phi^{j+}(\mathrm{x}, \mathrm{y}, \mathrm{z})-\phi^{j-}(\mathrm{x}, \mathrm{y}, \mathrm{z})=\bar{E}_{i}\left(\mathrm{x}_{i}^{j+}-x_{i}^{j-}\right) \quad(i=1,2,3)
$$

The application of B.C.s given in Eq. (3) and (4) satisfy the requirement of displacements and electrical potential of the unit cell periodicity and continuity.

The difference of the displacements for the corresponding points on the two opposite boundary surfaces should be specified due to the requirement of the Eq. (3). However, Li [49] derived an 
explicit displacement boundary conditions for unit cells representing microstructure of periodic pattern from the symmetry present in the microstructure. Extending Li [49] work and incorporating continuity of electric electrical charge, the complete set of B.Cs as required to obtain effective electromechanical properties of cellular materials are given in Table 2.

Table 2. Complete set of boundary conditions with computation formulae to determine electromechanical coefficients.

\begin{tabular}{|c|c|c|c|c|c|c|c|}
\hline Coefficients & $\mathbf{X}^{-}$ & $\mathbf{X}^{+}$ & $\mathbf{Y}^{-}$ & $\mathbf{Y}^{+}$ & $\mathbf{Z}^{-}$ & $\mathbf{Z}^{+}$ & Relation \\
\hline & $\bar{u}_{i} / \bar{\phi}$ & $\bar{u}_{i} / \bar{\phi}$ & $\bar{u}_{i} / \bar{\phi}$ & $\bar{u}_{i} / \bar{\phi}$ & $\bar{u}_{i} / \bar{\phi}$ & $\bar{u}_{i} / \bar{\phi}$ & \\
\hline $\bar{S}_{11}^{E}, \bar{S}_{12}^{E}$ & $0 / 0$ & $\bar{u}_{1} / 0$ & $0 / 0$ & $-/ 0$ & $0 / 0$ & $-/ 0$ & $\bar{\varepsilon}_{11} / \bar{\sigma}_{11}, \bar{\varepsilon}_{22} / \bar{\sigma}_{11}$, \\
$\bar{S}_{13}^{E}, \bar{d}_{31}^{E}$ & & & & & & & $\bar{\varepsilon}_{33} / \bar{\sigma}_{11}, \bar{D}_{3} / \bar{\sigma}_{11}$ \\
\hline $\bar{S}_{21}^{E}, \bar{S}_{22}^{E}$ & $0 / 0$ & $-/ 0$ & $0 / 0$ & $\bar{u}_{2} / 0$ & $0 / 0$ & $-/ 0$ & $\bar{\varepsilon}_{11} / \bar{\sigma}_{22}, \bar{\varepsilon}_{22} / \bar{\sigma}_{22}$, \\
$\bar{S}_{23}^{E}, \bar{d}_{32}^{E}$ & & & & & & & $\bar{\varepsilon}_{33} / \bar{\sigma}_{22}, \bar{D}_{3} / \bar{\sigma}_{22}$ \\
\hline $\bar{S}_{31}^{E}, \bar{S}_{32}^{E}$ & $0 / 0$ & $-/ 0$ & $0 / 0$ & $-/ 0$ & $0 / 0$ & $\bar{u}_{3} / 0$ & $\bar{\varepsilon}_{11} / \bar{\sigma}_{33}, \bar{\varepsilon}_{22} / \bar{\sigma}_{33}$, \\
$\bar{S}_{33}^{E}, \bar{d}_{33}^{E}$ & & & & & & & $\bar{\varepsilon}_{33} / \bar{\sigma}_{33}, \bar{D}_{3} / \bar{\sigma}_{33}$ \\
\hline $\bar{S}_{44}^{E}, \bar{d}_{24}^{E}$ & $\bar{u}_{2}=0$, & $\bar{u}_{2}=0$, & $\bar{u}_{1}=0$, & $\bar{u}_{1} \neq 0$, & $\bar{u}_{3}=0$ & $\bar{u}_{3}=0$ & $\bar{\varepsilon}_{12} / \bar{\sigma}_{12}$, \\
& $\bar{u}_{3}=0$ & $\bar{u}_{3}=0$ & $\bar{u}_{3}=0$ & $\bar{u}_{3}=0$ & $/ 0$ & $/ 0$ & $\bar{D}_{2} / \bar{\sigma}_{12}$ \\
\hline $\bar{S}_{55}^{E}, \bar{d}_{15}^{E}$ & $/ 0$ & $/ 0$ & $/ 0$ & $/ 0$ & & & \\
& $\bar{u}_{2}=0$, & $\bar{u}_{2}=0$, & $\bar{u}_{2}=0$, & $\bar{u}_{2}=0$, & $\bar{u}_{1}=0$, & $\bar{u}_{1} \neq 0$, & $\bar{\varepsilon}_{13} / \bar{\sigma}_{13}$, \\
& $\bar{u}_{3}=0$ & $\bar{u}_{3}=0$ & $/ 0$ & $/ 0$ & $\bar{u}_{2}=0$ & $\bar{u}_{2}=0$ & $\bar{D}_{1} / \bar{\sigma}_{13}$ \\
\hline $\bar{S}_{66}^{E}$ & $/ 0$ & $/ 0$ & & & $/ 0$ & $/ 0$ & \\
\hline $\bar{u}_{1}=0$, & $\bar{u}_{1}=0$, & $\bar{u}_{1}=0$, & $\bar{u}_{1}=0$, & $\bar{u}_{1}=0$, & $\bar{u}_{1}=0$, & $\bar{\varepsilon}_{23} / \bar{\sigma}_{23}$ \\
\hline $\bar{d}_{33}^{\sigma}, \bar{\kappa}_{33}^{\sigma}$ & $/ 0$ & $/ 0$ & $\bar{u}_{3}=0$ & $\bar{u}_{3}=0$ & $\bar{u}_{2}=0$ & $\bar{u}_{2} \neq 0$ & \\
\hline $\bar{d}_{15}^{\sigma}, \bar{\kappa}_{11}^{\sigma}$ & $* / 0$ & $-/ \bar{\phi}$ & $-/-$ & $-/-$ & $-/-$ & $-/-$ & $\bar{\varepsilon}_{13} / \bar{E}_{1}$, \\
& & & & & & & $\bar{D}_{1} / \bar{E}_{1}$ \\
\hline $\bar{d}_{24}^{\sigma}, \bar{\kappa}_{22}^{\sigma}$ & $-/-$ & $-/-$ & $* / 0$ & $-/ \bar{\phi}$ & $-/-$ & $-/-$ & $\bar{\varepsilon}_{12} / \bar{E}_{2}$, \\
& & & & & & & $\bar{D}_{2} / \bar{E}_{2}$ \\
\hline
\end{tabular}


*Points A, B and C are constrained on respective faces (having zero electric potential) to avoid rigid body motion.

\subsubsection{Homogenization Method}

, A homogenization procedure was adopted for micro-macro scale coupling to obtain effective properties for different macroscopic loading cases. The average stress and strain was obtained by volume (V) integration over the unit cell, such that:

$$
\bar{\sigma}_{i j}=\frac{1}{V} \int_{V} \sigma_{i j}(x, y, z) d V \quad \bar{\varepsilon}_{i j}=\frac{1}{V} \int_{V} \varepsilon_{i j}(x, y, z) d V
$$

Analogously the average electric fields and electrical displacements are defined by

$$
\bar{E}_{i}=\frac{1}{V} \int_{V} E_{i}(x, y, z) d V \quad \bar{D}_{i}=\frac{1}{V} \int_{V} D_{i}(x, y, z) d V
$$

Using traction continuity one can find that the average stress is represented by

$$
\bar{\sigma}_{i j}=\frac{R_{i j}}{A_{j}}(\text { no summation on } \mathrm{j})
$$

Using electrical charge continuity, the average electric displacement is represented by

$$
\bar{D}_{i}=\frac{q_{i}}{A_{i}}
$$

Eq. (7) demonstrates that the average stress over the unit cell can be simply obtained by dividing the resultant tractions $\left(R_{i j}\right)$ on the boundary surfaces from the areas $\left(A_{j}\right)$ of the corresponding boundary surfaces. Eq. 8 demonstrates that the average electrical displacement over the RVE can be simply obtained by dividing the resultant charge $\left(q_{i}\right)$ on the boundary surfaces from the areas ( $A_{i}$ ) of the corresponding boundary surfaces.

\section{Results and Discussion}


The mechanical and electrical loading conditions are applied to the unit cells as per the load cases shown in the Table 2 and subsequently the effective electromechanical properties are obtained. The detailed formulae for the computation of some of the typical electromechanical coefficients are also shown in Table 2. In each load case, the boundary conditions are applied to the unit cell as such that except one component of the effective stress and electric field vector in Eq. (2), all the remaining components would yield zero values. Results of these loading cases can yield 45 independent material parameters (including elastic, piezoelectric and dielectric parameters).

The effective components including the elastic compliance, $\bar{S}_{i j k l}^{E}$, piezoelectric strain tensors, $\left(\bar{d}_{k i j}^{E}, \bar{d}_{i k l}^{\sigma}\right)$, dielectric stress tensor $\bar{\kappa}_{i j}^{\sigma}$ with the corresponding elastic constants, $\bar{C}_{i j k l}^{E}$, elastic moduli, $\left(E_{i j}^{E}, \mathrm{G}_{k k}^{E}\right)$, and Poisson's ratio are obtained for each of the three piezoelectric metamaterial considered. The material constants are obtained for a range of angle of ligaments as well as for longitudinally poled and transversely poled systems. All the results are plotted in terms of $\theta$. It is because the angle $\theta$ controls the cellular topology and the levels of auxeticity of metamaterial.,. The letter LP and TP are associated with the results for the longitudinal and transverse poled cases, respectively. The following relations are used to obtain the constants to represent the behavior of PMs. 


$$
\begin{aligned}
& {\left[\boldsymbol{C}^{E}\right]=\left[\boldsymbol{S}^{E}\right]^{-1},} \\
& {[\boldsymbol{e}]=[\boldsymbol{d}]\left[\boldsymbol{C}^{E}\right],} \\
& {\left[\kappa^{\varepsilon}\right]=\left[\kappa^{\sigma}\right]-[d][\boldsymbol{e}]^{T},} \\
& {\left[\boldsymbol{S}^{D}\right]=\left[\boldsymbol{S}^{E}\right]-[\boldsymbol{d}]^{T}[\boldsymbol{g}],} \\
& {\left[\boldsymbol{C}^{D}\right]=\left[\boldsymbol{S}^{D}\right]^{-1},} \\
& {[\boldsymbol{g}]=[\boldsymbol{h}]\left[\boldsymbol{S}^{D}\right],} \\
& {[\boldsymbol{h}]=\left[\kappa^{\varepsilon}\right][\boldsymbol{e}] .}
\end{aligned}
$$

where $\left[\boldsymbol{C}^{D}\right],\left[\boldsymbol{S}^{D}\right],[\boldsymbol{e}],\left[\boldsymbol{\kappa}^{\varepsilon}\right],[\boldsymbol{g}],[\boldsymbol{h}]$ are the stiffness tensor for zero electric displacement, compliance tensor for zero electric displacement, piezoelectric stress tensor, dielectric strain tensor, piezoelectric voltage constants and piezoelectric strain tensor, respectively.

\subsection{Sanity Check}

A sanity check is performed to verify the boundary conditions. It is observed that an analytical model that compute the electromechanical properties of $\mathrm{HC}$ metamaterials based on orthotropic materials is yet not available in the literature. Therefore, the set of proposed boundary conditions as given in Table 2 are tested initially on one element and then on 100x100x100 elements cube for establishing the accuracy and suitability of the proposed method. It is found that the proposed boundary conditions yield the bulk properties of the PZT-5H as given in Table 1.

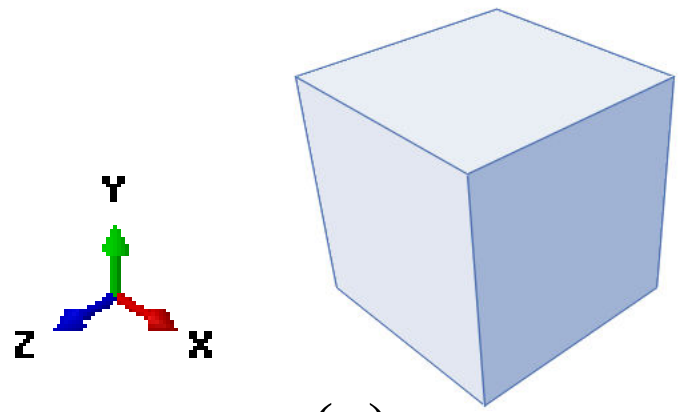

(a)

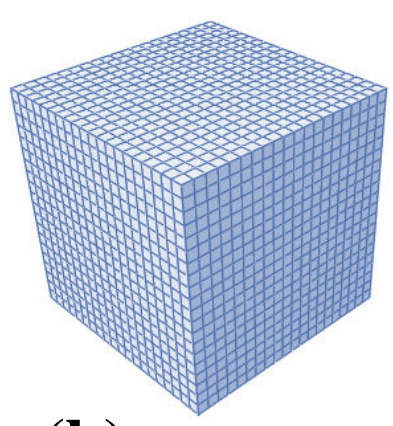

(b) 
Figure 3: Sanity Check with a) one element and b) 100x100x100 elements.

\subsection{Electromechanical Properties \\ 3.2.1.Effective Elastic Response}

The homogenization process is used to yield $\bar{S}_{i j k l}^{E}, \bar{d}_{i k l}^{\sigma}, \bar{\kappa}_{i j}^{\sigma}$, which are further utilized to compute other material constants. For example, the compliance matrix coefficients $\bar{S}_{i j k l}^{E}$ are used to compute the Young's moduli $E_{i j}^{E}$, shear moduli $G_{i j}^{E}$ and Poisson ratios $v_{i j}$. Figure 4 illustrates the Young's and shear moduli of longitudinally and transversely poled HC metamaterials over various angles $30^{\circ}, 45^{\circ}$ and $60^{\circ}$. Both longitudinally and transversely poled $\mathrm{HC}$ metamaterials has showed significant changes in both in-plane (x-y plane) and out-of-plane (z-axis) Young's moduli values for the different angles. This figure shows that the elastic properties are anisotropic and exhibit different trends.

The Young's moduli of the longitudinally and transversely poled HC metamaterials exhibits nonlinear dependence with the increase of the angle of the ligaments $(\theta)$. The piezoelectric REHC exhibits significantly enhanced Young's moduli $\mathrm{E}_{11}$ (same as $\mathrm{E}_{33}$ ) as compared to the C-HC and SRE-HC metamaterials. Whereas the C-HC metamaterials enhances Young's modulus $\mathrm{E}_{22}$. The lowest Young's modulus is observed for the transversely poled RE-HC. The effect of orthotropic ligament properties is prominent in the case of Young's moduli along y-axis. The lower values of $E_{22}$ are observed for the transversely poled as compared to the longitudinally poled systems due to higher constrained as provided by the electromechanical coupling parameters.

Among the Young's moduli values, the properties along y-axis (such as $\mathrm{E}_{22}$ ) of all the HC metamaterials are generally observed to be higher than the other moduli (such as $\mathrm{E}_{11}=\mathrm{E}_{33}$ ). This observation can be explained on the basis that the dominant deformation mode in the out-of-plane (y-axis directions) is axial, whereas the in-plane directions ( $\mathrm{x}$ or $\mathrm{z}$ directions) has showed 
bending/hinging dominated deformation mode. The highest Young's moduli isobserved for C-HC metamaterials in both poling directions.
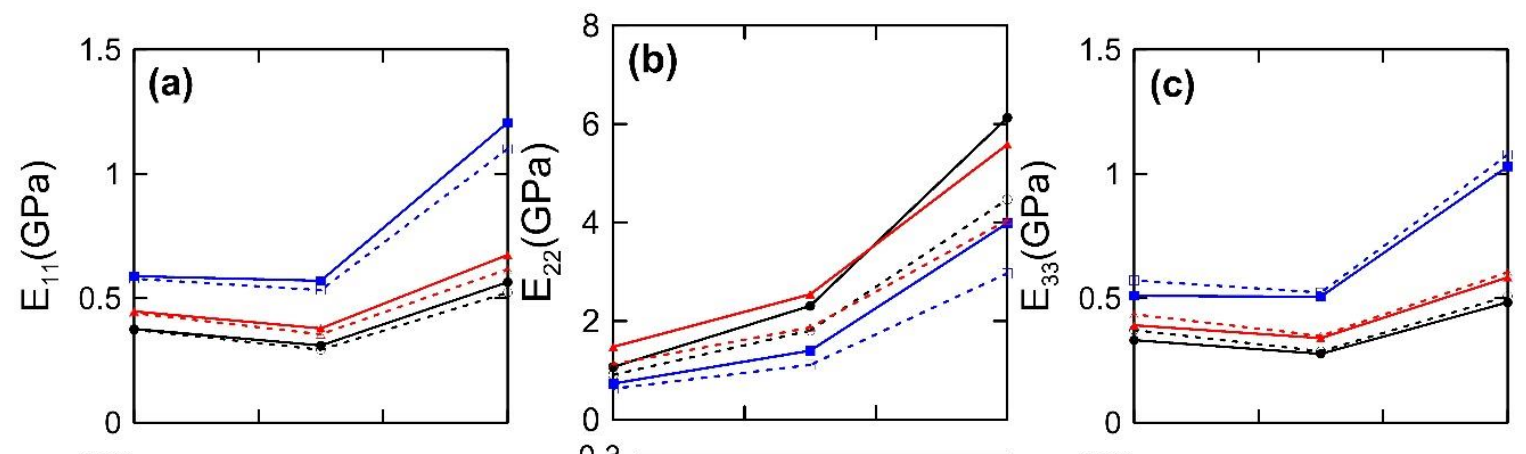

Configurations

$\bullet \bullet \mathrm{HC}(\mathrm{LP})$

๑๑- $\mathrm{HC}(\mathrm{TP})$

$\because-\rightarrow \mathrm{RE} \quad(\mathrm{LP})$

$\Xi-\Xi-\Xi R E \quad(T P)$

$\leadsto$ SRE (LP)

$\Delta-A-\triangle \operatorname{SRE}(\mathrm{TP})$
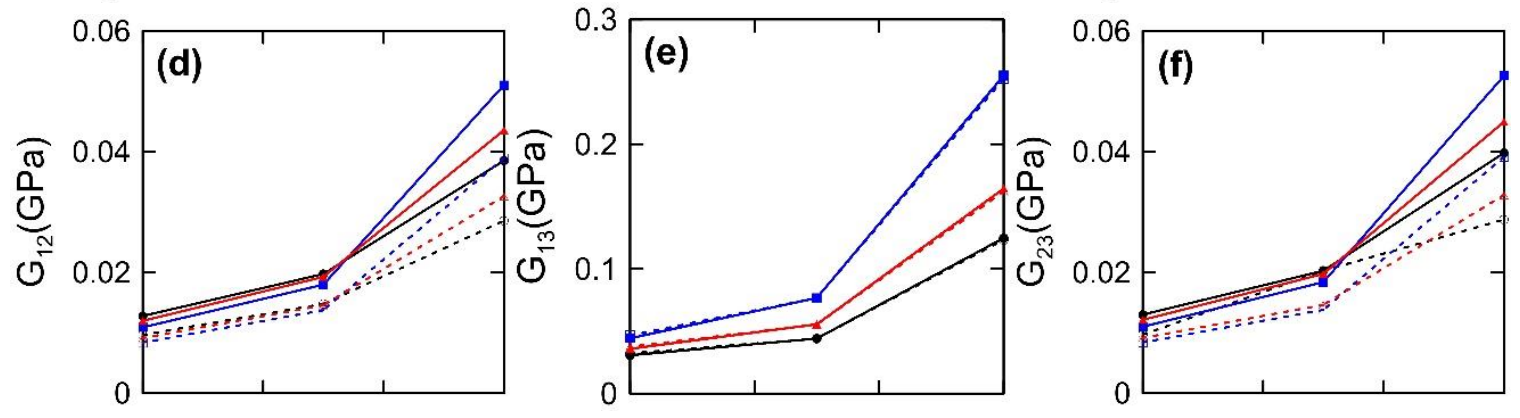

Figure 4: Variation in the overall elastic moduli with various angles $\left(30^{\circ}, 45^{\circ}, 60^{\circ}\right)$ in several classes of piezoelectric HC metamaterials. (a)-(c) Young's moduli and (d)-(f) Shear moduli.

The shear moduli along $\mathrm{x}$ - and $\mathrm{z}$ - axes (i.e., $\mathrm{G}_{12}=\mathrm{G}_{23}$ ) and along $\mathrm{y}$-direction (i.e., $\mathrm{G}_{13}$ ) has showed a nonlinear dependence on increasing angle of the ligaments $(\theta)$. This nonlinearity corresponds to the nonlinear relation of angle of the ligaments $(\theta)$ with the relative density. The dominance of the stiffness in the y-directions makes the UC stiffer under shear loading condition along 1-3 plane. In shear loading (2-1 and 2-3 planes), the dominant mode of deformation is bending in the struts and ligaments of the $\mathrm{HC}$ structures along one of the three directions. Among the three HC metamaterials, the 3D HC-RE are observed to have highest shear moduli (i.e., $\mathrm{G}_{13}$ ) both in longitudinally and transversely poled HC metamaterials.

The proposed HC metamaterials possesses wide range of Poison's ratios (positive, zero and negative). Their largest positive and negative Poisson's ratio has been observed at 45 degree as 
shown in Figure 5. These results illustrate that the HC based piezoelectric cellular materials can exhibit a wide range of combinations of elastic properties with different level of auxeticity. The Poisson's ratio due to loading in 2 direction $\left(v_{12}=v_{32}\right)$ are same due to the similar deformation in 1 and 3 directions. Similarly, due to similar deformation along Y-axis, the Poisson's ratio due to loading along $\mathrm{X}$-axis $\left(v_{21}\right)$ is same due to loading along $\mathrm{Y}$ - axis $\left(\mathrm{v}_{23}\right)$. The variety of Poisson's ratio can be used to create tunable transducers. However, the transducer with zero Poisson's ratio are excellent choice for axial actuators.
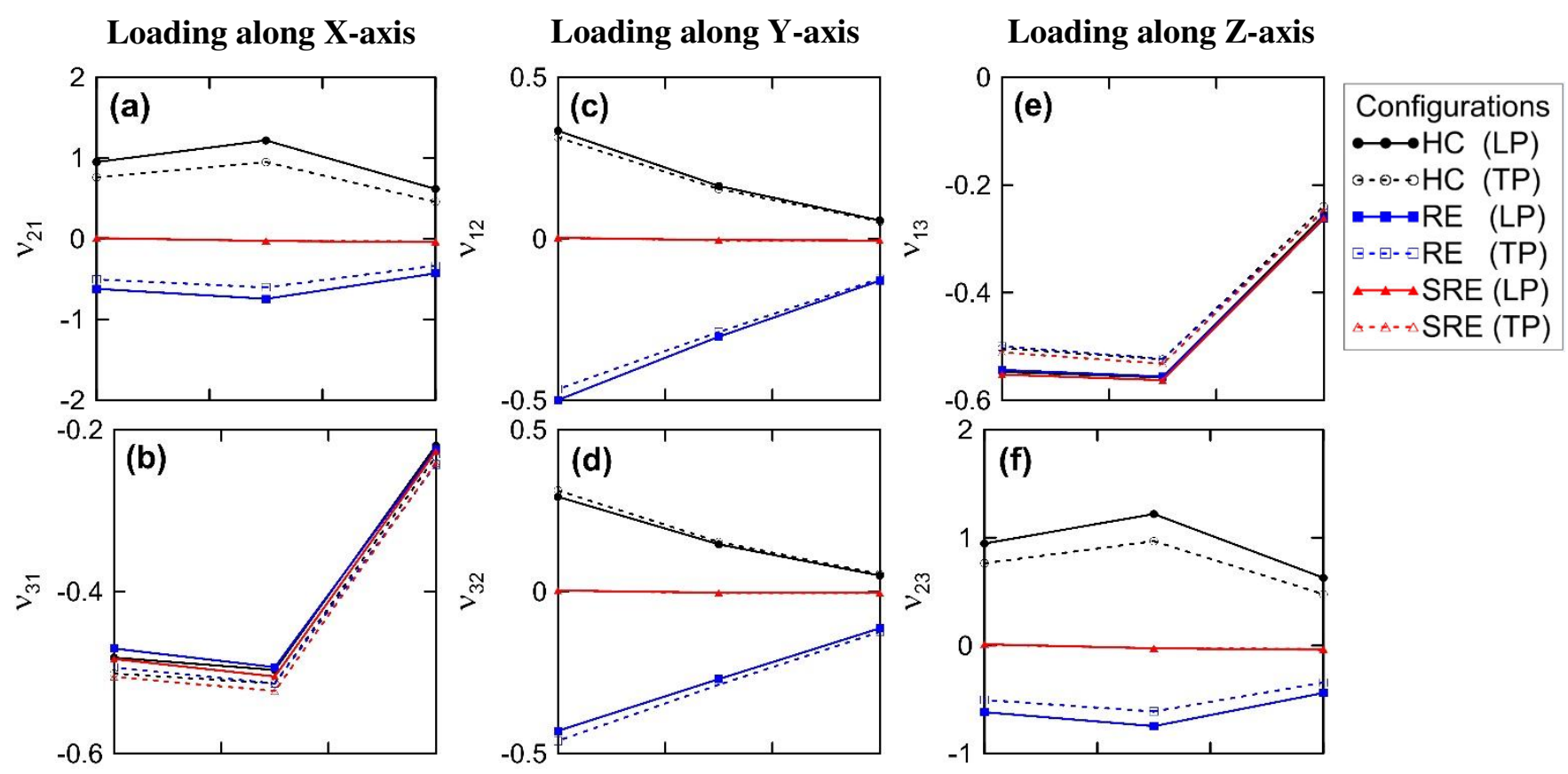

Figure 5: Variation in the overall Poisson's ratio with various angles $\left(30^{\circ}, 4^{\circ}, 6^{\circ}\right)$ in several classes of piezoelectric $\mathrm{HC}$ foam structures.

Figure 6 shows the contour plots of the field variables under different loadings. Figure 6(a) and (b) show the displacement contours for all the three HC structures in one-direction under uniaxial and shear loadings respectively.. These distribution of displacements clearly demonstrates the uniaxial and shear deformation states. Figure 6(c) shows the electric potential contours under 
electric field of $0.1 \mathrm{MV} / \mathrm{m}$. In all three cases, the electrical potential shows linear variation from one end to others with uniform electric potential at the central ligaments.

(a) Uniaxial Loading; $\varepsilon_{11}=0.1 \%$

(a) $3 \mathrm{D}-\mathrm{CHC}$
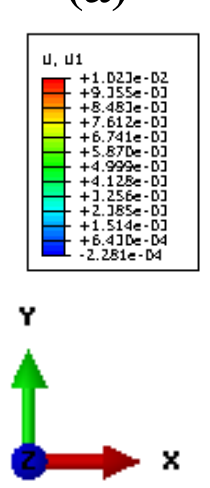

(b) $3 \mathrm{D}-\mathrm{CHC}$

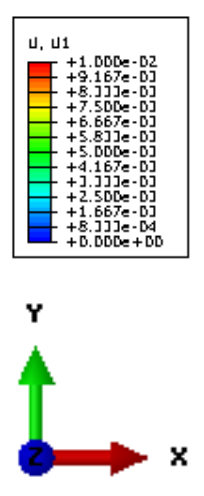

(c)
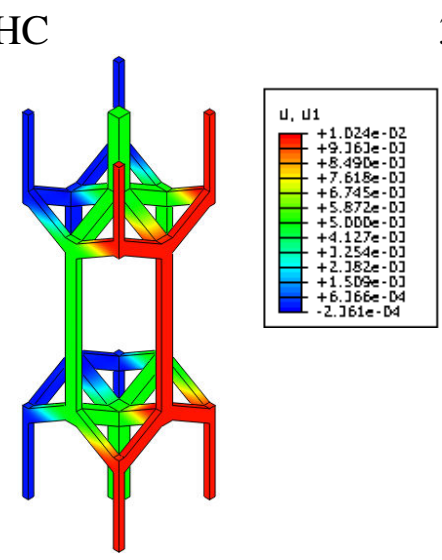

3D-RE

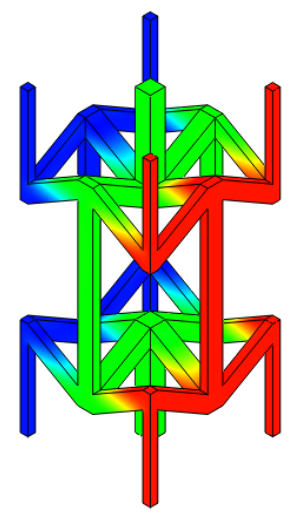

3D-SRE

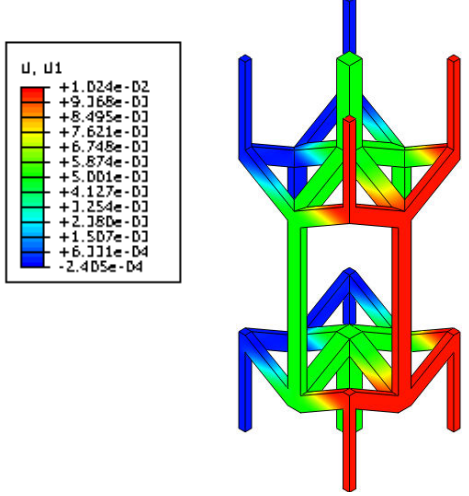

(b) Shear Loading; $\gamma_{12}=0.1 \%$

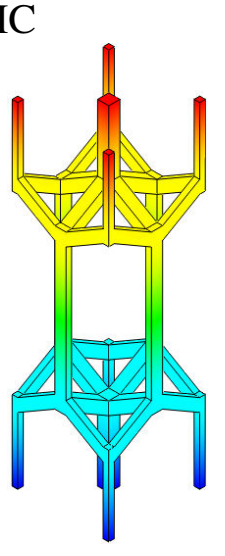

3D-RE

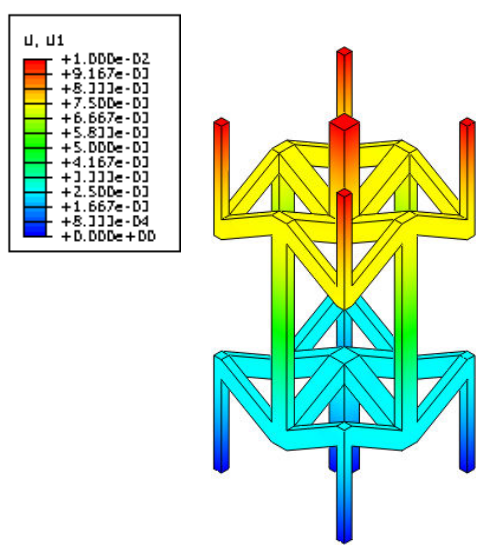

3D-SRE
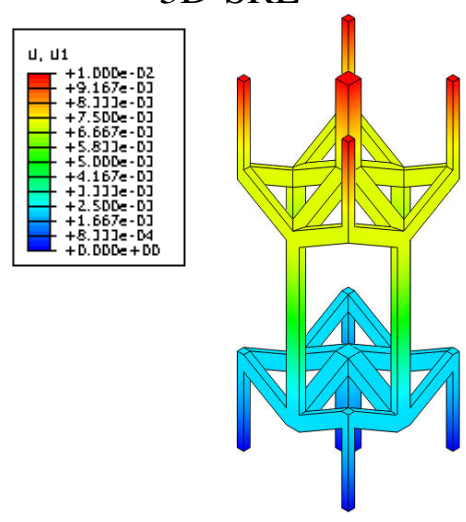

(c) Electrical Loading; $E_{1}=0.1 M V / m$
3D-CHC
3D-RE
3D-SRE 

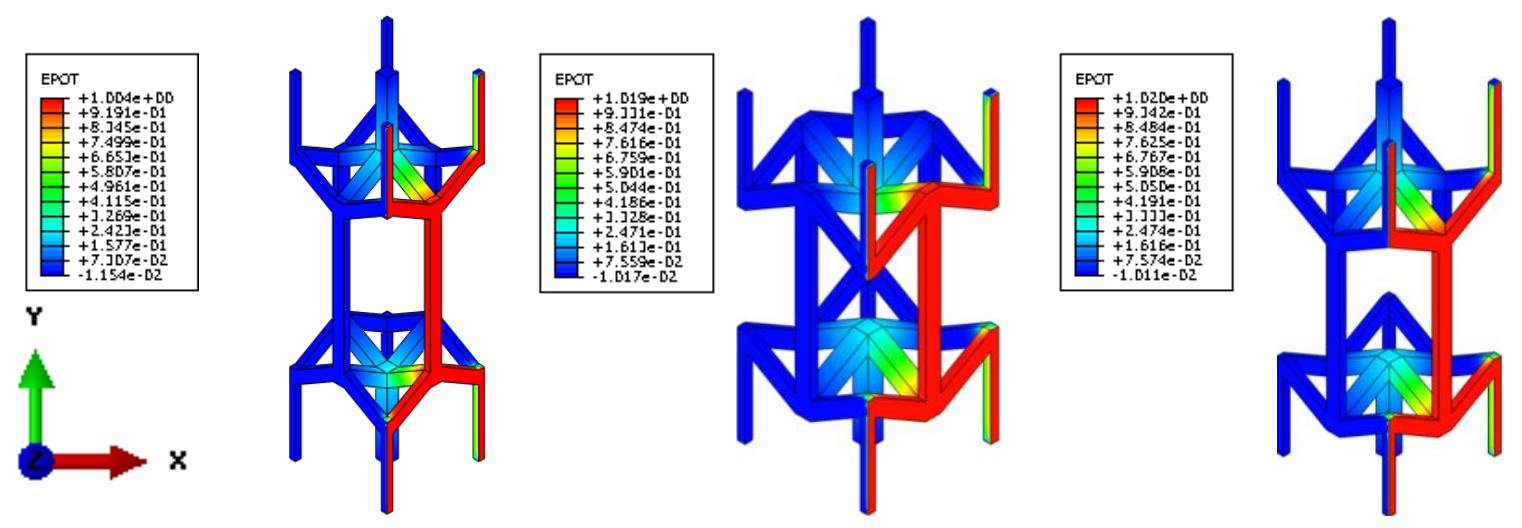

Figure 6 Displacement and electric potential contours in the unit cells of several classes of piezoelectric $\mathrm{HC}$ foam structures with angle $45^{\circ}$. a) Displacement contours under mechanical normal loading (i.e., $0.1 \%$ normal strain along the x-axis) b) Displacement contours under mechanical shear loading (i.e., $0.1 \%$ shear strain in the $x$-y plane). c) Electric potential contours under electric field of $0.1 \mathrm{MV} / \mathrm{m}$.

\subsubsection{Effective piezoelectric properties}

Figure 7 shows the variation in the normal- and shear-coupling-based piezoelectric properties with respect to ligament angles for the proposed HC metamaterials, In general, the piezoelectric properties of $\mathrm{HC}$ metamaterials are independent of the cellular network and the poling directions [18]. However, a significant dependence of the c topology is observed in some of the piezoelectric properties for both longitudinally and transversely poled systems. For transversely poled systems, the magnitude of constants is observed very less but its trends are observed similar to longitudinally poled systems .

It is also observed that $\mathrm{HC}$ metamaterials exhibits strong piezoelectric sensitivity to shear-type piezoelectric properties. However, the piezoelectric constants exhibits significantly different dependencies on $\theta$. For example, in longitudinally poled system $e_{24}$ (LP) increases the nonlinearly but $\mathrm{e}_{15}$ (LP) shows less increase in the values with $\theta$ for all cellular networks. For both $\mathrm{e}_{15}$ (LP) and $\mathrm{e}_{24}(\mathrm{LP})$, the largest values are observed at 60 degrees for 3D HC-RE.

The normal-based piezoelectric properties are also dependent on the topology and the angle $\theta$ of the ligaments. For bulk PMs, the normal piezoelectric properties along the poling directions, i.e., 
$\mathrm{e}_{33}$ (LP) and $\mathrm{e}_{33}(\mathrm{TP})$, are often considered the most important. In longitudinally poled system $\mathrm{e}_{33}$ (LP) shows very less variations with an increase in angle $\theta$ but it shows little variation for e33 (TP). The highest values of the normal piezoelectric properties are obtained for the 3D- CHC in longitudinally poled system. Figure 7 also shows the negative piezoelectric constants e32. The piezoelectric cellular material can exhibit positive $\mathbf{e}_{32}$ values but it dependson the angle $\theta$. Such behavior highlights that the HC cellular networks canexhibit a different crystal symmetry than that of the constituent material. Themaximum negative values of $e_{31}$ and $e_{32}$ are also observed for the longitudinally poled 3D HC-RE and CHC networks, respectively.

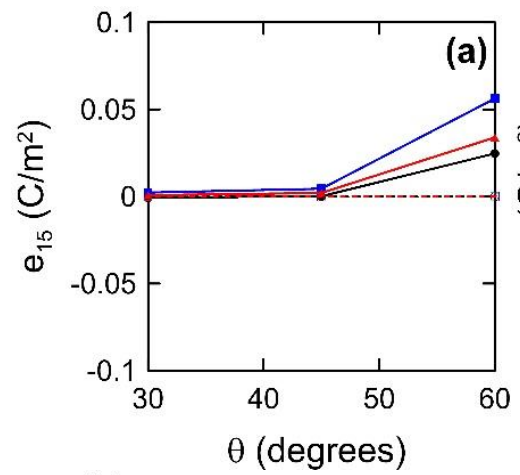

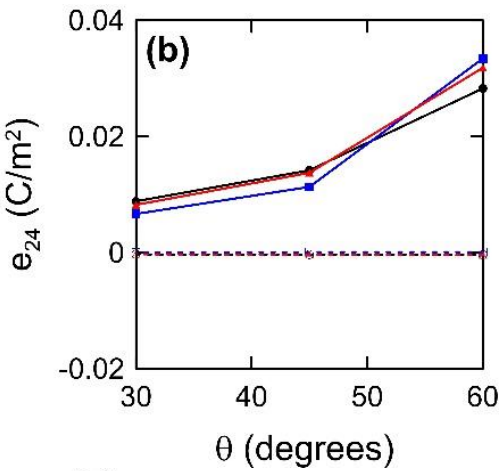

Configurations

$\bullet \bullet$ HC (LP)

๑-๑-॰HC (TP)

$\because R E \quad(L P)$

๑-घ- -RE (TP)

$\leadsto$ SRE (LP)

A-A- $\triangle$ SRE (TP)
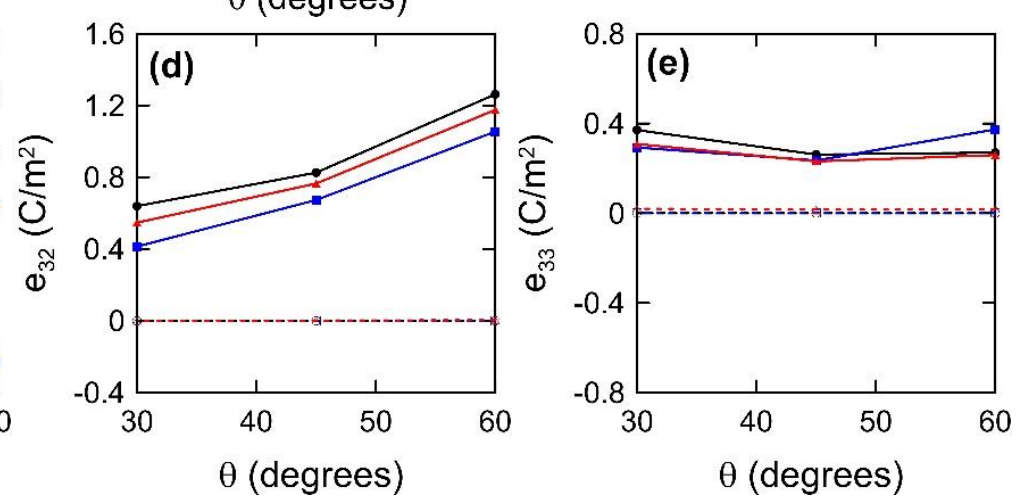

Figure 7: Variation in the overall piezoelectric constants with various angles $\left(30^{\circ}, 4^{\circ}, 6^{\circ}\right)$ in several classes of piezoelectric $\mathrm{HC}$ foam structures.

\subsubsection{Effective dielectric properties}

The effective dielectric properties $\kappa_{i j}$ under constant stress as a function of angle of the ligaments

$(\theta)$ is computed for all three HC metamaterials as shown in Figure 8. Both longitudinally and 
transversely poled HC metamaterials show significant changes in the dielectric constants values for different angles. The dielectric constants of all the considered HC networks exhibits nonlinear dependence and their values are increased with an increase in the angle of the ligaments $(\theta)$. The nonlinearity in the values of $\kappa_{i j}$ can be associated with the diverse path of the electric charges along the 3D complex networks of the ligaments. The highest and lowest values of the dielectric constants are observed for 3D HC-RE $\kappa_{22}(\mathrm{TP})$ and conventional HC $\kappa_{11}(\mathrm{LP})$ respectively.
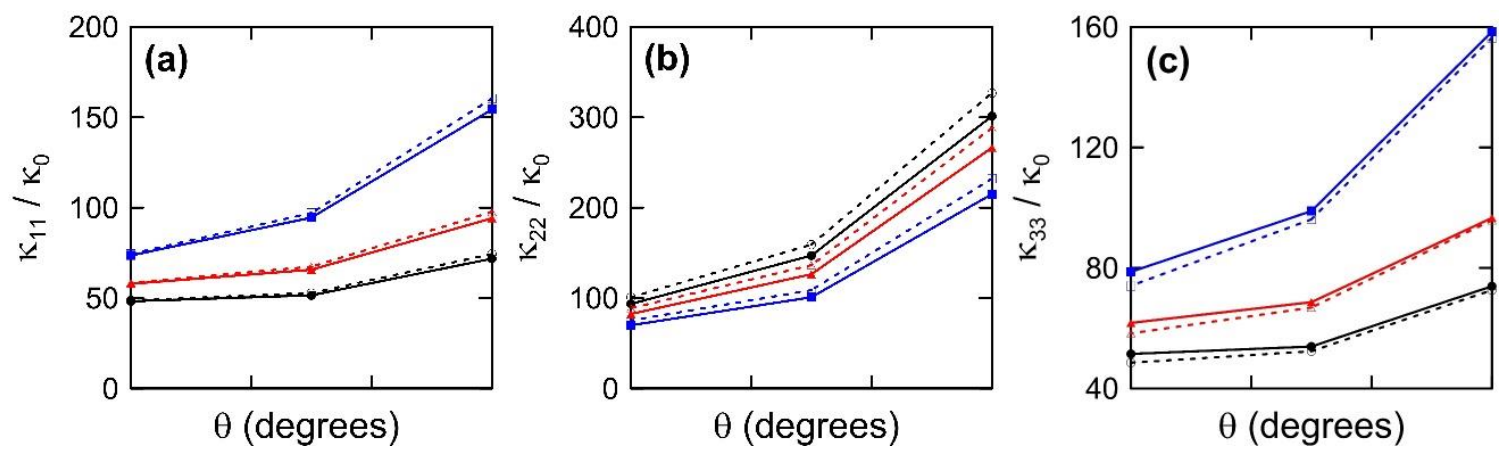

Configurations $\bullet \leftrightarrow \mathrm{HC}(\mathrm{LP})$ ๑-॰-॰HC (TP) $\because R E \quad(L P)$ ๑-घ- $-R E \quad(T P)$ $\longleftrightarrow$ SRE (LP) $\Delta-A-\triangle \operatorname{SRE}(\mathrm{TP})$

Figure 8: Variation in the overall dielectric constants with various angles $\left(30^{\circ}, 4^{\circ}, 6^{\circ}\right)$ in several classes of piezoelectric $\mathrm{HC}$ foam structures.

\section{Effective Figure of Merit}

The selection of the piezoelectric materials and their utility in various industrial applications, such as, ultrasound imager, hydrophones and energy harvesters are done based on the particular figure of merits (FOM). The combination of electromechanical constants are used to describe several industry-relevant FOM. For example, the constants that has direct relevance to FOM of cellular PMs and their potential applications (e.g., hydrophones) are the hydrostatic strain coefficient $\left(d_{h}\right.$ ), the hydrostatic figure of merit $\left(d_{h} \cdot \mathrm{g}_{h}\right)$,the acoustic impedance $(Z)$ and the electromechanical thickness model coupling factor $\left(k_{t}\right)$ ([29],[24]). In addition to these FOM, we have also presented several electro-elastic parameters (FOM) that are of interest in various applications. For more discussion on FOMs, please see [24].The list of parameters are given in Table 3. 
Table 3. List of figure of merit.

\begin{tabular}{|l|l|}
\hline Parameter & Relation \\
\hline hydrostatic strain coefficient $\left(d_{h}\right)$ & $d_{h}=d_{31}+d_{32}+d_{33}$ \\
\hline hydrostatic figure of merit $\left(d_{h} \cdot \mathrm{g}_{h}\right)$ & $g_{h} d_{h}$ with $g_{h}=g_{31}+g_{32}+g_{33}$ \\
\hline acoustic impedance $(Z)$ & $Z=\left[\rho C_{33}^{E}\right]^{1 / 2}$ \\
\hline $\begin{array}{l}\text { electromechanical thickness mode coupling } \\
\text { factor }\left(k_{t}\right)\end{array}$ & $k_{t}=\left[1-\frac{C_{33}^{E}}{C_{33}^{D}}\right]^{1 / 2}=\frac{e_{33}}{\left[C_{33}^{D} \kappa_{33}^{\varepsilon}\right]^{1 / 2}}$ \\
\hline electromechanical coupling factor $\left(k_{3 i}\right)$ & $\left.k_{3 i}=\frac{d_{3 i}}{\left[S_{i i}^{E} \kappa_{33}^{\sigma}\right.}\right]^{1 / 2} ;(\mathrm{i}=1,2$ with no sum on ii) \\
\hline Frequency constants $\left(N_{t}\right)$ & $N_{t}=\frac{1}{2}\left[\frac{C_{33}^{E}}{\rho}\right]^{1 / 2}$ \\
\hline Frequency constants $\left(N_{i}\right)$ & $N_{i}=\frac{1}{2}\left[\frac{1}{\rho S_{33}^{E}}\right]^{1 / 2} ;(\mathrm{i}=1,2$ with no sum on ii) \\
\hline
\end{tabular}

The computed figures of merit are first normalized with the corresponding figures of merit for the solid constituent material (PZT-5H) and then are plotted in Figure 8. All the normalized FOMs has not shown any enhanced response for transversely poled system except the acoustic impedance, Z. However, in longitudinally poled system, the normalized FOMs shows strong dependence on the angle $\theta$. The normalized hydrostatic strain coefficient shows very mild effect on the variation of angle $\theta$, while normalized hydrostatic figure of merit decreases significantly with an increase in the angle $\theta$. The maximum value is observed at 30 degrees. The large enhancement in the $d_{h} \cdot g_{h}$ is obtained due to the inverse relation between $\mathrm{g}_{33}$ and $\kappa_{33}$. Therefore, with an increase of porosity (reduction in angle $\theta$ ) can cause a decrease in the dielectric constant results an increase in $\mathrm{g}_{h}$ as shown in Figure 9. The normalized acoustic impedance is increased with an increase in the angle 
$\theta$. The best value (minimized) is observed at 45 degrees. These computational studies are agreed well with the experimental observations [14] and has showed a similar trend in the Z values with anincrease of porosity.

In comparison to their bulk constituent, all the piezoelectric $\mathrm{HC}$ networks exhibit an enhanced response especially for the longitudinal poling. Longitudinally poled networks exhibit four order of magnitude increase in their hydrostatic figure of merit. These enhancements are coupled with one orders of magnitude decrease in the acoustic impedance. Hydrostatic figure of merit and acoustic impedance are the two most important transducer parameters for the design of hydrophones. From the point of view of transducer design, the Z- $d_{h} \cdot \mathrm{g}_{h}$ relation is very important. Figure 9 shows the Z- $d_{h} \cdot \mathrm{g}_{h}$ tradeoff where, with the increase of porosity, an increase in the $d_{h} \cdot \mathrm{g}_{h}$ are accompanied by the decreases in acoustic impedance. This combination of increased sensitivity-based figures of merit and reduced acoustic impedance is unique, as it is impossible to realize using piezo-composites or bulk PMs [51]. Thus, it is possible to create piezoelectric 3D HC networks with the desirable electro-mechanical characteristics.
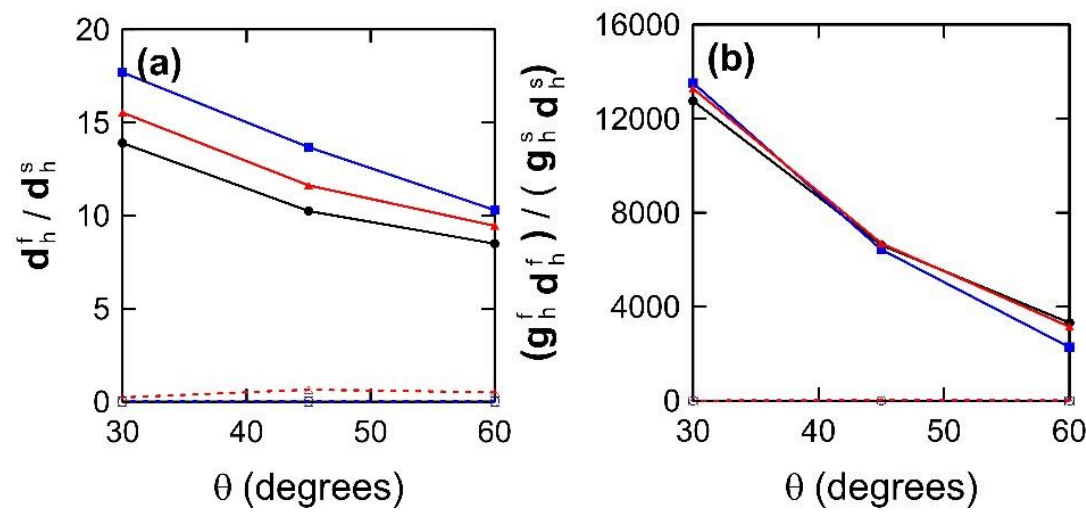

Configurations

$\bullet$ HC (LP) ๑-๑-॰HC (TP)

$\leadsto$ RE (LP)

-घ- $-\mathrm{RE} \quad(\mathrm{TP})$

$\leadsto$ SRE (LP)

$\triangle-A-\triangle$ SRE (TP) 

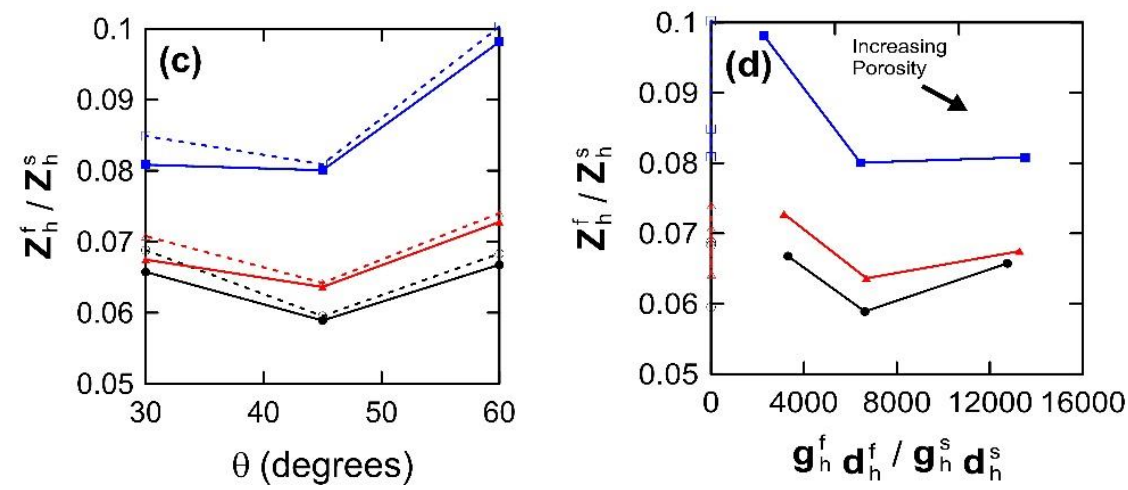

Figure 9: Variation in select normalized figures of merit with various angles in several classes of $\mathrm{HC}$ foam structures. (a) hydrostatic strain coefficient, (b) hydrostatic figure of merit, (c) acoustic impedance (d) Z- $d_{h} \cdot \mathrm{g}_{h}$ relation

Next, the normalized electromechanical coupling factors and frequency constants for the piezoelectric 3D HC networks are presented here. Figures 10(a-c) show the normalized magnitudes of the electromechanical coupling factors. Both the $\mathrm{k}_{31}$ and $\mathrm{k}_{32}$ show a very mild variation with the increase in angle $\theta$ and almost remain constant. For the bulk PZT-5H, both the electromechanical coupling factors $\mathrm{k}_{31}$ and $\mathrm{k}_{32}$ are computed as equal. However, for the $\mathrm{HC}$ cellular network, the electromechanical coupling factors $\mathrm{k}_{32}$ decrease more relative to $\mathrm{k}_{31}$ for a given angle of all $\mathrm{HC}$ networks because of the anisotropic nature of the microstructure. The $\mathrm{d}_{3 \mathrm{i}}$ are almost same for both the modes. But the larger values of $S_{22}^{E}$ than $S_{11}^{E}$ results in a larger decrease in $\mathrm{k}_{32}$ than $\mathrm{k}_{31}$. In contrast, to $\mathrm{k}_{31}$ and $\mathrm{k}_{32}$, the normalized thickness coupling factor, kt, are observedtwo order higher than the bulk PZT-5H.

Figures 10(d-f) show the normalized magnitudes of the electromechanical coupling factors. The frequency constants $\mathrm{N}_{1}$ and $\mathrm{N}_{2}$ are equal for the bulk PZT-5H. The frequency constant $\mathrm{N}_{2}$ has seen a decrease more relative to $\mathrm{N}_{1}$ for the given angles of all the $\mathrm{HC}$ networks. This is due to the orthotropic constituent material properties and the topology of the $\mathrm{HC}$ network relative to the bulk PZT-5H. Moreover, a reverse trend is observed for $\mathrm{N}_{1}$ and $\mathrm{N}_{2}$ with an increase in the porosity or 
the angle. This results is attributed to the larger values of $S_{22}^{E}$ than $S_{11}^{E}$ (and thus a larger decreased in $\mathrm{N}_{2}$ than $\mathrm{N}_{1}$ ). The thickness mode frequency constant $\mathrm{Nt}$ is increased with an increase in the angle. In other words, Nt is decreased with an increase of porosity as shown in Figure 10(f). The decrease in $\mathrm{N}_{\mathrm{t}}$ with an increase in the porosity can be associated with the increase in the $S_{33}^{E}$ due to decrease in the lateral damping.
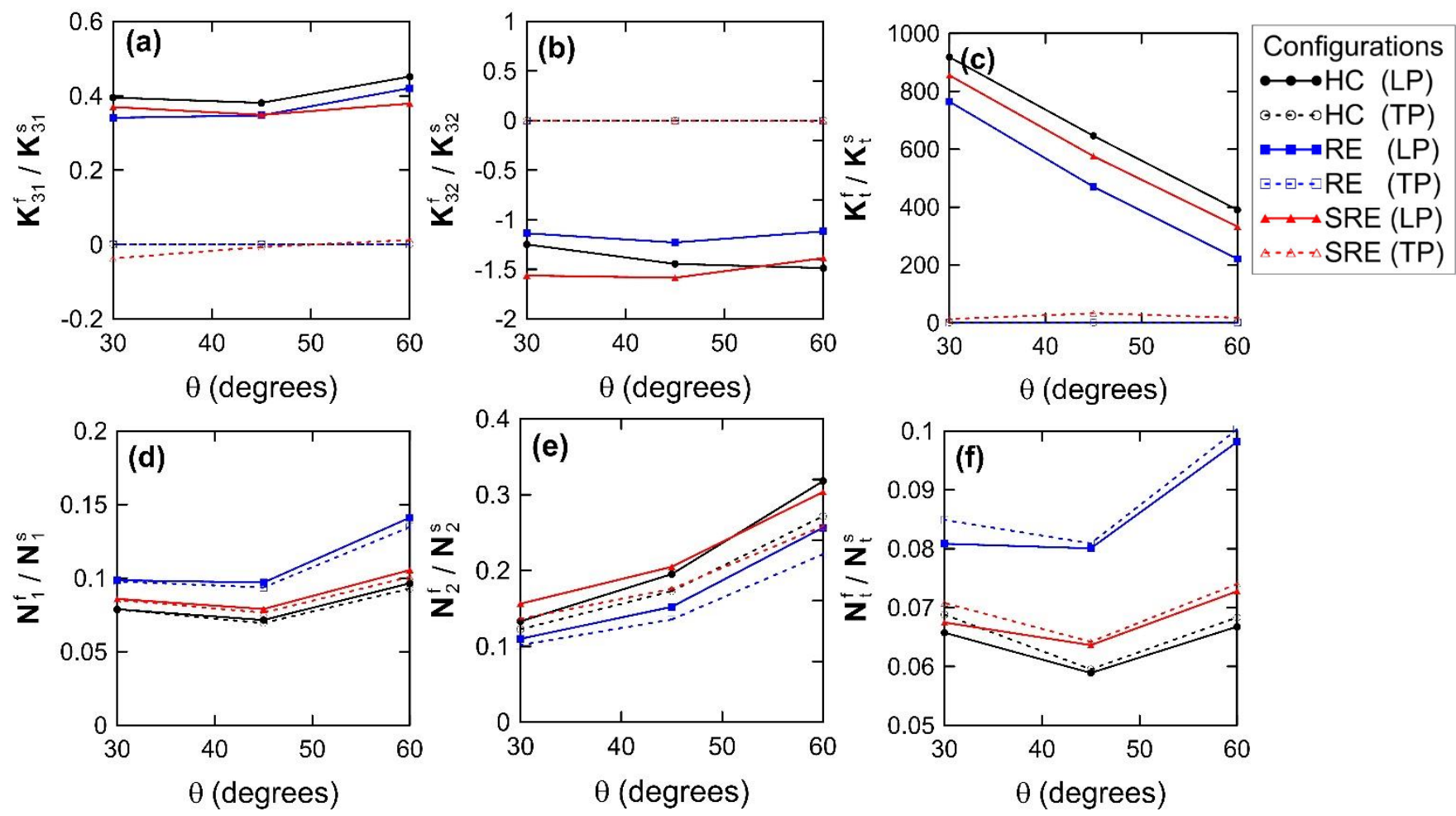

Figure 10: Variation in select normalized figures of merit with various angles in several classes of $\mathrm{HC}$ foam structures.

Overall, the mechanical and piezoelectric properties of the piezoelectric materials can be optimized by tailoring the architecture of the 3D-HC network and thus a unique combinations of tunable properties can be realized as required in various practical applications. The FE results show that the interplay between 3D-HC architecture and material anisotropy lead to a wide range of nonintuitive and architecture-dependent elastic, piezoelectric and dielectric properties that differ substantially from the properties of the architecture base material. The FE results have confirmed 
excellent electromechanical properties of 3D-CHC network. It is also confirms that the 3D-RE and 3D-SRE HC network exhibit a combination of excellent piezoelectric properties and unique mechanical properties (i.e. negative and zero Poisson's ratio) which cannot be obtained in 3DCHC network. The presented novel 3D-RE and 3D-SRE auxetic functional HC networks have the potential to design unique devices for sensing or actuating applications with negative and zero Poisson's ratio.

\section{Conclusions}

A micromechanical finite element approach is presented to compute the electromechanical properties of the 3-3 piezoelectric cellular metamaterials. A set of boundary conditions equivalent to periodic boundary conditions for periodic structure with certain symmetries are used to ensure that the displacements and electric potential of a unit cell are compatible across its boundaries with that of the adjacent unit cells. Three types of 3-3 piezoelectric HC cellular networks (conventional, auxetic and semi re-entrant) unit cells are analyzed using the proposed approach and its electromechanical properties are reported. Results show that for longitudinal poled system, the piezoelectric HC network exhibits a unique combination of properties, i.e., low impedance and more sensitivity, which can not be realized by bulk PMs. However, electromechanical properties of transversely poled system exhibit insignificant dependence on the ligament orientation and porosity. Results show that different type of auxetic behavior such as negative or zero Poisson's ratio can be realized and coupled with a wide range of tunable electromechanical characteristics. In the case of 3D-SRE, along with the highest out-of-plane modulus there is a configuration exists that yielded zero Pisson's ratio while loading along all three axes. The proposed 3D HC based metamaterials are good candidate for use as hydrophones as longitudinally poled networks exhibit four order of magnitude increase in their hydrostatic figure of merit and one order of magnitude 
decrease in the acoustic impedance. Amongst the three proposed HC networks, the 3D-RE exhibits the best overall combination of piezoelectric figures of merit.

\section{ACKNOWLEDEMENTS}

The authors acknowledge the help of Abdelrahman Alhammadi, Ali Alneyadi and Saeed Alqaydi from Khalifa University of Science and Technology in generating the parametric CAD files of the current honeycomb cellular networks. 


\section{REFERENCES}

[1] A. Muliana, Time dependent behavior of ferroelectric materials undergoing changes in their material properties with electric field and temperature, Int. J. Solids Struct. 48 (2011) 27182731. doi:10.1016/j.ijsolstr.2011.05.021.

[2] S. Marselli, V. Pavia, C. Galassi, E. Roncari, F. Craciun, G. Guidarelli, Porous piezoelectric ceramic hydrophone, J. Acoust. Soc. Am. 106 (1999) 733. doi:10.1121/1.427091.

[3] V.Y. Topolov, C.R. Bowen, Electromechanical properties in composites based on ferroelectrics, Springer Science \& Business Media, 2008.

https://www.google.com/books?hl=en\&lr=\&id=UPKTxPTp_hIC\&oi=fnd\&pg=PA1\&dq=E lectromechanical+Properties+in+Composite+Based+on+Ferroelectrics\&ots=Xh8b13Lk0\&sig=y-fV5t4RCgRGiMGMgm_Luu2s-w4 (accessed March 12, 2017).

[4] K. Hikita, K. Yamada, M. Nishioka, M. Ono, Piezoelectric properties of the porous PZT and the porous PZT composite with silicone rubber, Ferroelectrics. 49 (1983) 265-272. doi:10.1080/00150198308244698.

[5] W.A. Smith, The role of piezocomposites in ultrasonic transducers, in: Ultrason. Symp. 1989 Proc. IEEE 1989, IEEE, 1989: pp. 755-766.

http://ieeexplore.ieee.org/xpls/abs_all.jsp?arnumber=67088 (accessed August 12, 2016).

[6] S. Geis, P. Löbmann, S. Seifert, J. Fricke, Dielectric properties of PZT aerogels, Ferroelectrics. 241 (2000) 75-82.

[7] T. Arai, K. Ayusawa, H. Sato, T. Miyata, K. Kawamura, K. Kobayashi, Properties of hydrophone with porous piezoelectric ceramics, Jpn. J. Appl. Phys. 30 (1991) 2253.

[8] P. Guillaussier, C. Audoly, D. Boucher, Porous lead zirconate titanate ceramics for hydrophones, Ferroelectrics. 187 (1996) 121-128. doi:10.1080/00150199608244848.

[9] H. Kara, R. Ramesh, R. Stevens, C.R. Bowen, Porous PZT ceramics for receiving transducers, Ultrason. Ferroelectr. Freq. Control IEEE Trans. On. 50 (2003) 289-296.

[10] R.E. Newnham, D.P. Skinner, L.E. Cross, Connectivity and piezoelectric-pyroelectric composites, Mater. Res. Bull. 13 (1978) 525-536. doi:10.1016/0025-5408(78)90161-7.

[11] F. Levassort, M. Lethiecq, C. Millar, L. Pourcelot, Modeling of highly loaded 0-3 piezoelectric composites using a matrix method, Ultrason. Ferroelectr. Freq. Control IEEE Trans. On. 45 (1998) 1497-1505.

[12] M.J. Haun, R.E. Newnham, An experimental and theoretical study of 1-3 AND 1-3-0 piezoelectric PZT-Polymer composites for hydrophone applications, Ferroelectrics. 68 (1986) 123-139.

[13] J. Ueda, T.W. Secord, H.H. Asada, Large effective-strain piezoelectric actuators using nested cellular architecture with exponential strain amplification mechanisms, IEEEASME Trans. Mechatron. 15 (2010) 770-782.

[14] U. Bast, W. Wersing, The influence of internal voids with 3-1 connectivity on the properties of piezoelectric ceramics prepared by a new planar process, Ferroelectrics. 94 (1989) 229-242.

[15] W. Wirges, M. Wegener, O. Voronina, L. Zirkel, R. Gerhard-Multhaupt, Optimized Preparation of Elastically Soft, Highly Piezoelectric, Cellular Ferroelectrets from Nonvoided Poly(ethylene Terephthalate) Films, Adv. Funct. Mater. 17 (2007) 324-329. doi:10.1002/adfm.200600162. 
[16] S.-H. Lee, S.-H. Jun, H.-E. Kim, Y.-H. Koh, Fabrication of Porous PZT-PZN Piezoelectric Ceramics With High Hydrostatic Figure of Merits Using Camphene-Based Freeze Casting, J. Am. Ceram. Soc. 90 (2007) 2807-2813. doi:10.1111/j.1551-2916.2007.01834.x.

[17] K. Nagata, H. Igarashi, K. Okazaki, R.C. Bradt, Properties of an Interconnected Porous $\mathrm{Pb}(\mathrm{Zr}, \mathrm{Ti}) \mathrm{O}_{3}$ Ceramic, Jpn. J. Appl. Phys. 19 (1980) L37-L40. doi:10.1143/JJAP.19.L37.

[18] S. Iyer, M. Alkhader, T.A. Venkatesh, Electromechanical behavior of auxetic piezoelectric cellular solids, Scr. Mater. 99 (2015) 65-68.

[19] K.S. Challagulla, T.A. Venkatesh, Electromechanical response of piezoelectric foams, Acta Mater. 60 (2012) 2111-2127.

[20] J. Bauer, S. Hengsbach, I. Tesari, R. Schwaiger, O. Kraft, High-strength cellular ceramic composites with 3D microarchitecture, Proc. Natl. Acad. Sci. 111 (2014) 2453-2458. doi:10.1073/pnas.1315147111.

[21] P. Fang, M. Wegener, W. Wirges, R. Gerhard, L. Zirkel, Cellular polyethylene-naphthalate ferroelectrets: Foaming in supercritical carbon dioxide, structural and electrical preparation, and resulting piezoelectricity, Appl. Phys. Lett. 90 (2007) 192908.

[22] H.N. Wadley, Multifunctional periodic cellular metals, Philos. Trans. R. Soc. Lond. Math. Phys. Eng. Sci. 364 (2006) 31-68.

[23] H. Banno, Theoretical equations for dielectric and piezoelectric properties of ferroelectric composites based on modified cubes model, Jpn. J. Appl. Phys. 24 (1985) 445.

[24] M.L. Dunn, M. Taya, Electromechanical Properties of Porous Piezoelectric Ceramics, J. Am. Ceram. Soc. 76 (1993) 1697-1706. doi:10.1111/j.1151-2916.1993.tb06637.x.

[25] Y. Mikata, Explicit determination of piezoelectric Eshelby tensors for a spheroidal inclusion, Int. J. Solids Struct. 38 (2001) 7045-7063. doi:10.1016/S0020-7683(00)00419-4.

[26] C.R. Bowen, V.Y. Topolov, Piezoelectric sensitivity of PbTiO 3-based ceramic/polymer composites with 0-3 and 3-3 connectivity, Acta Mater. 51 (2003) 4965-4976.

[27] R. Kar-Gupta, T.A. Venkatesh, Electromechanical response of piezoelectric composites: Effects of geometric connectivity and grain size, Acta Mater. 56 (2008) 3810-3823.

[28] P.W. Bosse, K.S. Challagulla, T.A. Venkatesh, Effects of foam shape and porosity aspect ratio on the electromechanical properties of 3-3 piezoelectric foams, Acta Mater. 60 (2012) 6464-6475.

[29] R. Kar-Gupta, T.A. Venkatesh, Electromechanical response of porous piezoelectric materials, Acta Mater. 54 (2006) 4063-4078.

[30] J.N. Grima, L. Oliveri, D. Attard, B. Ellul, R. Gatt, G. Cicala, G. Recca, Hexagonal honeycombs with zero Poisson's ratios and enhanced stiffness, Adv. Eng. Mater. 12 (2010) 855-862.

[31] I.G. Masters, K.E. Evans, Models for the elastic deformation of honeycombs, Compos. Struct. 35 (1996) 403-422.

[32] L.J. Gibson, M.F. Ashby, Cellular solids: structure and properties, Cambridge university press, 1997.

[33] H.X. Zhu, J.F. Knott, N.J. Mills, Analysis of the elastic properties of open-cell foams with tetrakaidecahedral cells, J. Mech. Phys. Solids. 45 (1997) 319-343.

[34] M. Alkhader, M. Vural, The partition of elastic strain energy in solid foams and lattice structures, Acta Mater. 57 (2009) 2429-2439.

[35] S.D. Papka, S. Kyriakides, Biaxial crushing of honeycombs:-Part 1: Experiments, Int. J. Solids Struct. 36 (1999) 4367-4396. 
[36] T. Fey, F. Eichhorn, G. Han, K. Ebert, M. Wegener, A. Roosen, Ken-ichi Kakimoto, P. Greil, Mechanical and electrical strain response of a piezoelectric auxetic PZT lattice structure, Smart Mater. Struct. 25 (2016) 015017. doi:10.1088/0964-1726/25/1/015017.

[37] K.A. Khan, R.K. Abu Al-Rub, Time dependent response of architectured Neovius foams, Int. J. Mech. Sci. 126 (2017) 106-119. doi:10.1016/j.ijmecsci.2017.03.017.

[38] L. Yang, O. Harrysson, H. West, D. Cormier, Mechanical properties of 3D re-entrant honeycomb auxetic structures realized via additive manufacturing, Int. J. Solids Struct. (2015). http://www.sciencedirect.com/science/article/pii/S0020768315002152 (accessed October 31, 2015).

[39] J. Yang, An Introduction to the Theory of Piezoelectricity, Springer Science \& Business Media, 2006.

[40] M.H. Luxner, J. Stampfl, H.E. Pettermann, Finite element modeling concepts and linear analyses of 3D regular open cell structures, J. Mater. Sci. 40 (2005) 5859-5866. doi:10.1007/s10853-005-5020-y.

[41] T. Kanit, S. Forest, I. Galliet, V. Mounoury, D. Jeulin, Determination of the size of the representative volume element for random composites: statistical and numerical approach, Int. J. Solids Struct. 40 (2003) 3647-3679.

[42] K.A. Khan, A.H. Muliana, A multi-scale model for coupled heat conduction and deformations of viscoelastic functionally graded materials, Compos. Part B Eng. 40 (2009) 511-521. doi:10.1016/j.compositesb.2009.02.003.

[43] M. Jiang, I. Jasiuk, M. Ostoja-Starzewski, Apparent thermal conductivity of periodic twodimensional composites, Comput. Mater. Sci. 25 (2002) 329-338.

[44] D.W. Abueidda, A.S. Dalaq, R.K. Abu Al-Rub, H.A. Younes, Finite element predictions of effective multifunctional properties of interpenetrating phase composites with novel triply periodic solid shell architectured reinforcements, Int. J. Mech. Sci. 92 (2015) 80-89. doi:10.1016/j.ijmecsci.2014.12.004.

[45] T.I. Zohdi, P. Wriggers, eds., An Introduction to Computational Micromechanics, Springer Berlin Heidelberg, Berlin, Heidelberg, 2005. http://link.springer.com/10.1007/978-3-54032360-0 (accessed November 2, 2015).

[46] Z. Xia, Y. Zhang, F. Ellyin, A unified periodical boundary conditions for representative volume elements of composites and applications, Int. J. Solids Struct. 40 (2003) 19071921. doi:10.1016/S0020-7683(03)00024-6.

[47] S. Iyer, T.A. Venkatesh, Electromechanical response of porous piezoelectric materials: Effects of porosity connectivity, Appl. Phys. Lett. 97 (2010) 072904.

[48] S. Iyer, T.A. Venkatesh, Electromechanical response of (3-0) porous piezoelectric materials: Effects of porosity shape, J. Appl. Phys. 110 (2011) 034109.

[49] S. Li, Boundary conditions for unit cells from periodic microstructures and their implications, Compos. Sci. Technol. 68 (2008) 1962-1974.

[50] J. Zhang, M.F. Ashby, The out-of-plane properties of honeycombs, Int. J. Mech. Sci. 34 (1992) 475-489. doi:10.1016/0020-7403(92)90013-7.

[51] M.L. Dunn, M. Taya, Micromechanics predictions of the effective electroelastic moduli of piezoelectric composites, Int. J. Solids Struct. 30 (1993) 161-175. 
2018-12-18

3-3 piezoelectric metamaterial with negative and zero Poisson's ratio for hydrophones applications

Khan, Kamran Ahmed

Elsevier

Khan KA, Khan M. (2019) 3-3 piezoelectric metamaterial with negative and zero Poisson's ratio for hydrophones applications. Materials Research Bulletin, Volume 112, April 2019, pp.194-204 https://doi.org/10.1016/j.materresbull.2018.12.016

Downloaded from Cranfield Library Services E-Repository 